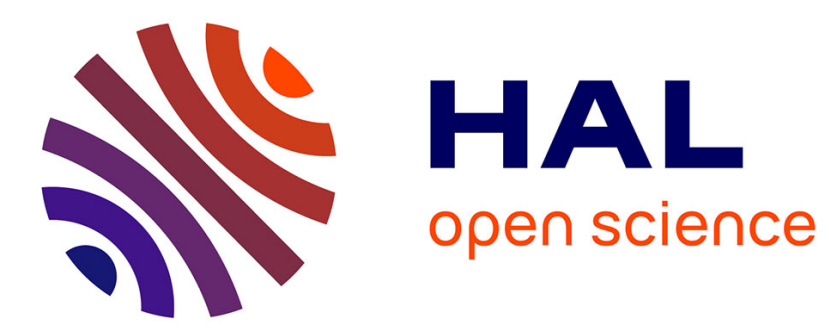

\title{
Biochemical composition and energy content of size-fractionated zooplankton east of the Kerguelen Islands
}

Mireille Harmelin-Vivien, Daniela Bănaru, Charlotte R Dromard, Melanie Ourgaud, Francois Carlotti

\section{To cite this version:}

Mireille Harmelin-Vivien, Daniela Bănaru, Charlotte R Dromard, Melanie Ourgaud, Francois Carlotti. Biochemical composition and energy content of size-fractionated zooplankton east of the Kerguelen Islands. Polar Biology, 2019, 42 (3), pp.603-617. 10.1007/s00300-019-02458-8 . hal-02053856

\section{HAL Id: hal-02053856 https://hal.science/hal-02053856}

Submitted on 1 Mar 2019

HAL is a multi-disciplinary open access archive for the deposit and dissemination of scientific research documents, whether they are published or not. The documents may come from teaching and research institutions in France or abroad, or from public or private research centers.
L'archive ouverte pluridisciplinaire HAL, est destinée au dépôt et à la diffusion de documents scientifiques de niveau recherche, publiés ou non, émanant des établissements d'enseignement et de recherche français ou étrangers, des laboratoires publics ou privés. 


\section{Biochemical composition and} energy content of size-fractionated zooplankton east of the Kerguelen Islands

Mireille Harmelin-Vivien,

Phone (+33) 486090637

Email mireille.harmelin@mio.osupytheas.fr

Daniela Bănaru,

Email daniela.banaru@mio.osupytheas.fr

Charlotte R. Dromard, 2

Email charlotte.dromard@univ-antilles.fr

Mélanie Ourgaud, 
Emailmelanie.ourgaud@mio.osupytheas.fr

François Carlotti, 1

Emailfrancois.carlotti@mio.osupytheas.fr

1 Aix Marseille Université, Université de Toulon, CNRS, IRD, Mediterranean Institute of Oceanography (MIO), UM

110, 13288 Marseille Cedex 09, France

2 UMR BOREA, CNRS 7208-MNHN-Sorbonne Université-UCN-IRD 207-UA, Laboratoire D’Excellence « CORAIL », Université Des Antilles, 97157 Pointe-à-Pitre, Guadeloupe

\section{Received: 14 July 2018 / Accepted: 5 January 2019}

\section{Abstract}

Food quality is recognized as a key parameter of food web functioning in which zooplankton plays a crucial role not only in linking lower to upper trophic levels but also in transforming the quality of the organic matter available to predators. The influence of size and taxonomic group composition of zooplankton in these processes was assessed in eastern Kerguelen waters (Southern Ocean) at the onset of the spring bloom in 2011. Biochemical (lipids, proteins and carbohydrates) and elemental (carbon and nitrogen) composition were measured in five size-fractions of bulk zooplankton ranging from $80 \mu \mathrm{m}$ to $>2000 \mu \mathrm{m}$ and in large copepods, euphausiids, annelids and salps, and energy content was derived from biochemical contents. Proteins were the dominant component of zooplankton dry weight $(21.5 \% \mathrm{dw})$, followed by lipids $(8.9 \% \mathrm{dw})$, soluble carbohydrates $(2.2 \% \mathrm{dw})$ and insoluble carbohydrates $(1.0 \% \mathrm{dw})$. A concentration increase with zooplankton size for all biochemical components was observed, particularly stronger for proteins and lipids. Copepods and salps provided, respectively, the highest and the lowest amount of lipids and energy. A four-fold increase in energy content was observed from the smallest to the largest fraction inducing a significant increase (> 
$10 \mathrm{~kJ} \mathrm{~g}^{-1} \mathrm{dw}$ ) in the quality of zooplankton matter. This may explain why large zooplankton represent a major food resource for numerous fish, seabirds and marine mammals in the Southern Ocean. Such unique results are required to better quantify energy dynamics in polar food webs.

\section{Keywords}

Southern Ocean

Plankton

Lipids

Proteins

Carbohydrates

Energy content

\section{Introduction}

Zooplankton plays a central role in ecosystem functioning and food webs in transforming and transferring the organic matter from lower (phytoplankton sensu lato) to higher trophic levels (zooplanktivorous carnivores) up to large predators (Banse 1995; Frederiksen et al. 2006). In the Southern Ocean, zooplankton constitutes a huge biomass of prey consumed not only by small planktivorous fishes such as myctophids, melamphaids and gempylids (Pakhomov et al. 1996; Cherel et al. 2010) but also by a number of seabirds and marine mammals (Cherel et al. 2002, 2005). The Kerguelen Islands support large colonies of landbased seabirds, seals and elephant seals that forage directly on zooplankton or zooplanktivorous consumers (Guinet et al. 1996; Bocher et al. 2002; Hindell et al. 2011). Meso- and macrozooplankton copepods, euphausiids and amphipods may each represent up to 50 $-80 \%$ of the food ingested by different species of diving seabirds, such as petrels and penguins (Ridoux 1994; Bocher et al. 2001; Cherel et al. 2005).

Many studies have underlined the importance of considering predator size - prey size relationships to describe properly the length, trophic structure and functioning of food webs, and to predict their dynamics and evolution under the climate change (Barnes et al. 2010; Blanchard 
et al. 2017). In pelagic ecosystems, predator-prey size relationships are also of major importance within planktonic communities among species and developmental stages of organisms, particularly for enhancing biomass trophic transfer to consumers (Hansen et al. 1994; GarciaComas et al. 2016). If zooplankton were previously considered as a single functioning entity in early food web models, size-based groups of zooplanktonic organisms are now entered in ecosystem modeling (Carlotti et al. 2000; Travers-Trolet et al. 2007). Three groups of zooplankton based on taxonomy, trophic level and maximum size were included in an ecosystem model of the Kerguelen Islands waters (Pruvost et al. 2005). But size is not the only important parameter. To ensure a true understanding of planktonic food webs, it is necessary to consider the abundance, biomass and composition of the different size fractions of zooplankton, along with the environmental drivers of their population dynamics (Carlotti et al. 2015).

In addition, the knowledge of the biochemical composition of zooplankton size fractions may enable us to approximate the energy density of an environment, the efficiency of organic matter transfer along food webs and the energy amount that the high-level predators may obtain when feeding on one size fraction or another. Many studies have been published on the elemental or biochemical composition of zooplankton (see Donnelli et al. 1994; Postel et al. 2000; Hagen and Auel 2001; among others). However, most of them were performed either on bulk zooplankton without information on species and size composition (Bhat et al. 1993; Nageswara Rao and Ratna Kumari 2002; Arun Kumar et al. 2013), or on the dominant species (Percy and Fife 1981; Reinardt and van Vleet 1986; Torres et al. 1994). Much information is available on lipid content, particularly for polar species (Ward et al. 1996; Falk-Petersen et al. 1999; Lee et al. 2006; Mayzaud et al. 2011), but less for proteins, carbohydrates or energy content (Donnelly et al. 1993; Färber-Lorda et al. 2009; Arun Kumar et al. 2013).

AQ1

The aim of our study was thus to provide comprehensive information on the biochemical (lipids, proteins and carbohydrates) and elemental (C and $\mathrm{N}$ ) compositions, and energy content (joules), of zooplankton size- 
based fractions at different stations east of Kerguelen Islands during early phytoplankton spring bloom. Our more specific objectives were: (1) to determine the variations of zooplankton biochemical and elemental compositions according to fraction size and stations (2) to relate these variations to the broad composition by taxonomic groups of fractions and to environmental parameters (temperature, salinity and chlorophyll $a$ concentration), and (3) to quantify the energy content of zooplankton in eastern Kerguelen waters.

\section{Material and methods}

\section{Study site and sampling}

The naturally iron-fertilized region of the Kerguelen Islands is characterized by a complex mesoscale structure of water masses with contrasted biogeochemical characteristics, generating highphytoplankton and zooplankton biomass (Blain et al. 2008; Carlotti et al. 2015). The polar front (PF), defined as the northern most boundary of the winter water colder than $2{ }^{\circ} \mathrm{C}$, passes above the plateau south of the Kerguelen Islands and then follows northwards the eastern shelf break before turning eastwards and southwards in a meandering course (Fig. 1), separating the cold Antarctic Surface Water (AASW) in the south from the warm Subantarctic Surface Water (SASW) in the north (Park et al. 2014; Pauthenet et al. 2018), and inducing the mixing of Feenriched shelf waters in the oceanic waters east of the archipelago (Trull et al. 2015). Located between the PF in the south and the Subantarctic front (SAF) in the north, the Kerguelen Islands are bathed by the polar frontal surface water (PFSW) (Park et al. 2014). Sampling of zooplankton was performed in early austral spring (15 October-20 November 2011) during the Kerguelen Ocean and Plateau Compared Study cruise II (KEOPS2 survey) at 13 stations east of the Kerguelen Islands (Fig. 1). Most stations were located in AASW south of the meandering polar front. One station (R2) was located in the deep water south-west of the Kerguelen Islands in high-nutrients low-chlorophyll (HNLC) waters, two stations (A3 and E-4W) were located over the Kerguelen plateau, and seven stations (TNS-7, E-1, E-2, E-3, E-4E, E-5 and TEW-4) in the northernmost AASW in a quasi-stationary meander of the PF. Two other stations (F-L and TEW-8) were located in deep 
PFSW at the extreme north-east of the study region (Park et al. 2014). Station A3 was sampled twice, in pre-spring bloom (A3-1) and spring bloom conditions (A3-2). The six stations E (E-1 to E-5) corresponded to a quasi-Lagrangian survey of a stationary meander of the PF over a 20-day period during which the same water mass was sampled repeatedly. Detailed information on sampling strategy, hydrodynamic conditions and plankton communities during the KEOPS2 survey were given in Carlotti et al. (2015) and Trull et al. (2015). Zooplankton was collected with a double Bongo (60 $\mathrm{cm}$ mouth diameter) with one $330 \mu \mathrm{m}$ and one $120 \mu \mathrm{m}$ mesh nets mounted with filtering cod ends. For biochemical analyzes, one haul was performed at each station from $250 \mathrm{~m}$ depth to the surface at a speed of $0.5 \mathrm{~m} \mathrm{~s}^{-1}$ during the day (Carlotti et al. 2015). Samples were filtered through five sieves arranged in a column $(2000,1000,500,200$ and $80 \mu \mathrm{m}$ mesh size $)$ to obtain five size-based fractions $(80-200 \mu \mathrm{m}, 200-500 \mu \mathrm{m}, 500$ $-1000 \mu \mathrm{m}, 1000-2000 \mu \mathrm{m}$ and $>2000 \mu \mathrm{m})$. When abundant, some large organisms from the fraction $>2000 \mu \mathrm{m}$ were sorted by broad taxonomic groups (copepods, euphausiids, annelids and salps) and kept separately. All samples were then frozen at $-20{ }^{\circ} \mathrm{C}$ until analysis.

\section{Fig. 1}

Localization of stations analyzed for biochemical and elemental composition of plankton east of the Kerguelen Islands during the KEOPS2 survey, with position of the polar front and water masses (modified from Park et al. 2014). AASW Antarctic surface water, PFSW polar frontal surface water, $S A S W$ subantarctic surface water 


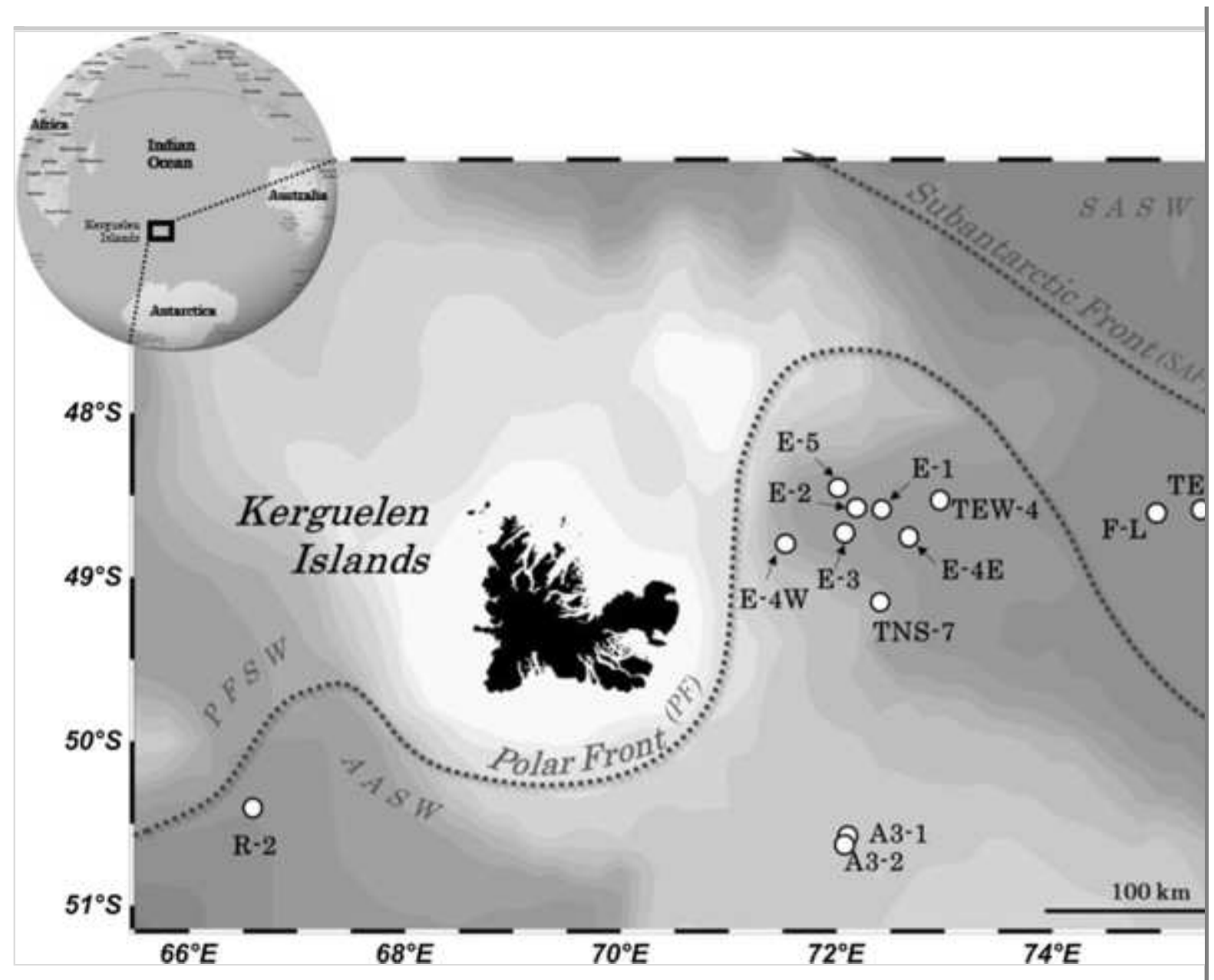

Differences in elemental and biochemical composition of zooplankton among stations were related to some environmental parameters of water masses: temperature $\left(T,{ }^{\circ} \mathrm{C}\right)$ and salinity (S) of surface water (Park et al. 2014; Trull et al. 2015), and vertically integrated chlorophyll $a$ concentration ( $\mathrm{Chl} a, \mathrm{mg} \mathrm{m}^{-2}$ ) from the surface to $200 \mathrm{~m}$ (Lasbleiz et al. 2014), along with the chemometric characteristics of phytoplankton accounting for level of iron fertilization, phytoplankton biomass and growth rate as defined by Trull et al. (2015), and further referred to as T-groups. T-group 1 (stations R2, TNS-7 and TEW-4) and T-group 2 (stations E-1, E-2, E-3, E4-E and E-5) were characterized by low phytoplankton biomass, and low to moderate iron supply and growth rate. T-group 3 (A3-1, A3-2 and E-4W), located over the plateau, were characterized by moderate to high phytoplankton biomass and iron supply, and high growth rate, while T-group 5 (TEW-8 and F-L), located downstream the PF in Subantarctic mode waters, presented high phytoplankton biomass, moderate to high iron supply and moderate 
growth (see Trull et al. 2015 for more details). T-group 4 corresponded to coastal stations not sampled for biochemical analyzes.

\section{Biochemical and elemental analyzes}

Before processing, identification of the main taxonomic groups in each fraction was performed under a binocular microscope and the relative importance of the different groups was visually estimated. Then, samples were freeze-dried and ground into a homogeneous fine powder with an agate mortar and pestle. The biochemical analyzes of lipids (LIP), proteins (PRO), and soluble (SC) and insoluble (IC) carbohydrates were determined by spectrophotometric methods. Lipids were measured following the method of Bligh and Dyer (1959), modified by Mayzaud and Martin (1975), and expressed as tripalmitic acid equivalent. Protein concentrations were determined according to the method of Lowry et al. (1951) and expressed as bovine seroalbumin equivalent. Soluble carbohydrates were extracted with distilled hot water $\left(100^{\circ} \mathrm{C}, 20 \mathrm{mn}\right)$ and insoluble carbohydrates in the residue. Both soluble and insoluble carbohydrates were determined by a modified method of Dubois et al. (1956), and expressed as glucose equivalents. Elemental analyzes of carbon $(\% \mathrm{C})$ and nitrogen $(\% \mathrm{~N})$ were obtained using the elemental analyzer (Flash EA1112 Thermo Scientific, Milan, Italy) during stable isotope measurements (see Carlotti et al. 2015). Three replicates were run on well-homogenized material for each type of analysis. Thus, at each station, 12 to 15 analyzes were done for each biochemical or elemental component ( 4 or 5 size classes $\times 3$ replicates). All concentrations $\left(\mathrm{mg} \mathrm{g}^{-1}\right)$ and percentages $(\%)$ were expressed on a dry weight basis (dw).

\section{Energy content estimation}

The energy value (Ei) of each size fraction in kilo Joules per gram dw of plankton was estimated using the equation (Eq. 1):

$E i_{\text {Fraction }}=\left[\left(C_{\text {Lip }} \times 35.6\right)+\left(C_{\text {Pro }} \times 21.4\right)+\left(C_{\text {SolCarb }} \times 17.2\right)+\left(C_{I n}\right.\right.$

where $C_{\mathrm{Lip}}=$ concentration of lipids in the fraction i in $\mathrm{mg} \mathrm{g}^{-1} \mathrm{dw}, C_{\mathrm{Pro}}=$ concentration of proteins in the fraction $\mathrm{i}$ in $\mathrm{mg} \mathrm{g}^{-1} \mathrm{dw}, C_{\text {SolCarb }}=$ concentration of soluble carbohydrates in the fraction i in $\mathrm{mg} \mathrm{g}^{-1} \mathrm{dw}$, 
$C_{\text {InsCarb }}=$ concentration of insoluble carbohydrates in the fraction $\mathrm{i}$ in $\mathrm{mg}$ $\mathrm{g}^{-1} \mathrm{dw}$. The coefficients 35.6, 21.4 and 17.2 are the conversion factor in Joules recommended for lipids, proteins and carbohydrates, respectively, by Postel et al. (2000) for zooplankton, following Beukema (1997).

The total amount of energy provided by zooplankton per square meter per station $\left(E_{\mathrm{T}} \mathrm{kJ} \mathrm{m}^{-2}\right)$ in the 0-250 $\mathrm{m}$ water column was calculated as the sum of the biomass ( $\mathrm{Bi}$ in $\mathrm{g} \mathrm{m}^{-2} \mathrm{dw}$ ) of each size fraction at this station multiplied by its energy value Ei ( $\mathrm{kJ} \mathrm{g}^{-1}$ ) (Eq. 2), for the 4 fractions $>200 \mu \mathrm{m}$ :

$$
E_{\mathrm{T}}=\sum(\mathrm{Bi} \times \mathrm{Ei})
$$

Biomasses $\left(\mathrm{g} \mathrm{m}^{-2} \mathrm{dw}\right)$ of the four larger zooplankton size fractions at each station were extracted from data given by Carlotti et al. (2015). The lowest plankton size fractions $(80-200 \mu \mathrm{m})$ were not taken into account in energy calculation, as they were mainly composed of phytoplankton.

\section{Data analyzes}

After testing for normality and homogeneity of variances, the effect of size was tested following one-way ANOVA or non-parametric Kruskal -Wallis tests followed by appropriate paired comparison tests. Relationships between elemental $(\% \mathrm{C}, \% \mathrm{~N}$ and $\mathrm{C} / \mathrm{N})$ and proximal $(\%$ LIP, \%PRO, \%SC and \%IC) components were tested by Pearson's linear correlations. A redundancy analysis (RDA) was used to relate the biochemical characteristics of plankton (biochemical concentrations, energy, $\% \mathrm{C}$ and $\% \mathrm{~N}$ ) to environmental and morphological factors (station, date, T, $\mathrm{S}, \mathrm{Chl} a$, T-group and size). RDA is a constrained ordination analysis and represents multivariate data in a reduced number of axes of the greatest variability (Legendre and Legendre 2012). As variables were expressed in different units $\left(\mathrm{mg} \mathrm{g}^{-1}, \mathrm{~kJ} \mathrm{~g} \mathrm{~g}^{-1}\right.$, $\%$ ), the analysis was done on transformed reduced centered data. $F$ tests based on 999 permutations were used to test the significance of RDA axes, the global effect of the environment (all variables taken into 
account) and the effect of each environmental variable. The forward selection of RDA was used to determine the minimum number of explanatory factors that could explain significant proportions of variation in biochemical data. This RDA analysis was done using the software R (package 'vegan').

\section{Results}

\section{Biochemical composition and energy value of size fractions and groups}

Proteins were the dominant component of zooplankton, representing a mean percentage of $\mathrm{x} \pm \mathrm{SD}=21.5 \pm 8.4 \% \mathrm{dw}, n=186$, followed by lipids $(8.9 \pm 4.4 \% \mathrm{dw}, n=186)$. Soluble carbohydrates $(2.2 \pm 0.6 \% \mathrm{dw}$, $n=186)$ and insoluble carbohydrates $(1.0 \pm 0.4 \% \mathrm{dw}, n=186)$ presented much lower percentages. If we considered only the sum of the biochemical components extracted, and not the total dry weight, proteins formed $63.1 \pm 4.3 \%$ of organic components extracted, lipids $26.8 \pm 2.9 \%$ and carbohydrates $(\mathrm{SC}+\mathrm{IC}) 10.1 \pm 2.9 \%$. The $\% \mathrm{C}$ and $\% \mathrm{~N}$ ranged from 22.5 to $53 \% \mathrm{dw}$ (mean $41.6 \pm 13.1 \% \mathrm{dw}$ ) and from 4.5 to $8.4 \% \mathrm{dw}$ (mean $7.1 \pm 2.0 \% \mathrm{dw}$ ), respectively, from the smallest to the largest size fractions. The total amount of biochemical components extracted increased from $15.9 \pm 7.6 \% \mathrm{dw}$ in the smallest fraction to 41.2 $\pm 6.9 \%$ in the largest one. Inversely, the proportion of residue, composed of ashes and not recovered components, decreased from 84.1 $\pm 7.6 \% \mathrm{dw}$ in fraction $80-200 \mu \mathrm{m}$ to $58.8 \pm 6.9 \% \mathrm{dw}$ in fraction $>2000 \mu \mathrm{m}($ mean $=66.4 \pm 12.2 \% \mathrm{dw})$. The two lowest size fractions (80-200 $\mu \mathrm{m}$ and 200-500 $\mu \mathrm{m}$ ) presented generally the lowest and the most different concentration values of all components (Table 1). They were also the most variable as indicated by higher coefficients of variation ( $\mathrm{CV}$, not shown) ( $\mathrm{CV}=45$ as a mean for fraction $80-200 \mu \mathrm{m}$ and only 20 for fraction $>2000 \mu \mathrm{m})$. Among the biochemical components, LIP and IC presented the most variable concentrations in all size fractions ( $\mathrm{CV}=39$ for both), followed by PRO $(\mathrm{CV}=2-8)$, while $\mathrm{SC}$ were the least variable within and between fractions $(\mathrm{CV}=21)$. For all components, except SC, mean concentrations did not differ between the three larger fractions $(500-1000 \mu \mathrm{m}, 1000-2000 \mu \mathrm{m}$ and $>2000 \mu \mathrm{m}$ ), as indicated by the results of post hoc comparisons (Table 1). PRO and LIP concentrations increased more with size $(\times 2.9$ 
and $\times 2.7$, respectively, between the lowest and the largest fractions), than SC and IC $(\times 1.7$ and $\times 1.4$, respectively). The energy content of zooplankton fractions was strongly correlated to LIP concentration (Pearson linear regression, $r=0.92, p<0.001, n=186$ ), as lipids were the most energy-rich components, and secondarily with PRO ( $r=0.89$, $p<0.001, n=186$ ), which were the dominant components in zooplankton. Accordingly, the energy content of zooplankton fractions increased with increasing fraction size (Table 1). The energy content of zooplankton during KEOPS2 cruise increased from a mean of $3.9 \mathrm{~kJ} \mathrm{~g}^{-1}$ $\mathrm{dw}$ in the fraction $80-200 \mu \mathrm{m}$ (from 2.1 to $7.9 \mathrm{~kJ} \mathrm{~g}^{-1} \mathrm{dw}$ at stations E-1 and $\mathrm{R} 2$, respectively) to a mean of $10.4 \mathrm{~kJ} \mathrm{~g}^{-1} \mathrm{dw}$ in the fraction $>2000 \mu \mathrm{m}$ (from 7.8 to $13.6 \mathrm{~kJ} \mathrm{~g}^{-1} \mathrm{dw}$ at stations E1 and E5, respectively). Percentages of organic $\mathrm{C}$ and nitrogen, and $\mathrm{C} / \mathrm{N}$ ratio also significantly increased with fraction size (Table 1). The $\mathrm{C} / \mathrm{N}$ ratio was not significantly different between the two smallest fractions (mean \pm $\mathrm{SD}=4.9 \pm 0.6, n=36$, and $5.5 \pm 0.7, n=39$, in the $80-200 \mu \mathrm{m}$ and 200 $-500 \mu \mathrm{m}$ fractions, respectively). In the largest fractions, $\mathrm{C} / \mathrm{N}$ increased from $6.1 \pm 0.9, n=39$, in the $500-1000 \mu \mathrm{m}$ fraction to $6.6 \pm 1.6, n=36$, in the $>2000 \mu \mathrm{m}$ fraction, but the difference was not significant. Significant linear correlations were observed between $\% \mathrm{C}, \% \mathrm{~N}$ and $\mathrm{C} / \mathrm{N}$ ratio, and all biochemical components and energy values in zooplankton fractions (Table 2). Both $\% \mathrm{C}$ and $\% \mathrm{~N}$ were best correlated with proteins, while $\mathrm{C} / \mathrm{N}$ was best correlated with lipids, and energy content with $\% \mathrm{C}$.

\section{Table 1}

Mean ( \pm standard deviation) concentrations of lipids (LIP), proteins (PRO), solul and insoluble carbohydrates (IC), percentages of organic carbon $(\% \mathrm{C})$ and nitro content (E) in size fractionated zooplankton (0-250 m) of Kerguelen during the KF

\begin{tabular}{|c|c|c|c|c|c|c|}
\hline $\begin{array}{l}\text { Size } \\
\text { fraction } \\
(\mu \mathrm{m})\end{array}$ & $n$ & $\begin{array}{l}\text { LIPa } \\
\left(\mathrm{mg} \mathrm{g}^{-1}\right. \\
\mathrm{dw})\end{array}$ & $\begin{array}{l}\operatorname{PRO}^{\mathrm{a}} \\
\left(\mathrm{mg} \mathrm{g}^{-1} \mathrm{dw}\right)\end{array}$ & $\begin{array}{l}\mathrm{SC}^{\mathrm{a}} \\
\left(\mathrm{mg} \mathrm{g}^{-1} \mathrm{dw}\right)\end{array}$ & $\begin{array}{l}I^{b} \\
\left(\mathrm{mg} \mathrm{g}^{-1} \mathrm{dw}\right)\end{array}$ & $\begin{array}{l}\% C^{b} \\
d w\end{array}$ \\
\hline $80-200$ & 36 & $\begin{array}{l}46.43 \pm \\
22.09^{c}\end{array}$ & $\begin{array}{l}89.08 \pm \\
54.13^{c}\end{array}$ & $\begin{array}{l}14.78 \pm \\
4.97^{\mathrm{c}}\end{array}$ & $8.44 \pm 3.81^{\mathrm{a}}$ & \\
\hline
\end{tabular}

Results of post-hoc tests performed on statistical analyses are indicated by lette indicating statistically significant differences. $n=$ number of analyses

${ }^{\mathrm{a} O n e-w a y ~ A N O V A}(F)$, Tuckey post hoc test

${ }^{b}$ Kruskal-Wallis ANOVA $(H)$, post hoc paired comparison test 


\begin{tabular}{|c|c|c|c|c|c|c|}
\hline $\begin{array}{l}\text { Size } \\
\text { fraction } \\
(\mu \mathrm{m})\end{array}$ & $n$ & $\begin{array}{l}\mathrm{LIP}^{\mathrm{a}} \\
\left(\mathrm{mg} \mathrm{g}^{-1}\right. \\
\mathrm{dw})\end{array}$ & $\begin{array}{l}\mathrm{PRO}^{\mathrm{a}} \\
\left(\mathrm{mg} \mathrm{g}^{-1} \mathrm{dw}\right)\end{array}$ & $\begin{array}{l}\mathrm{SC}^{\mathrm{a}} \\
\left(\mathrm{mg} \mathrm{g}^{-1} \mathrm{dw}\right)\end{array}$ & $\begin{array}{l}I^{b} \\
\left(\mathrm{mg} \mathrm{g}^{-1} \mathrm{dw}\right)\end{array}$ & $\begin{array}{l}\% C^{b} \\
d w\end{array}$ \\
\hline & & & & & & $\begin{array}{l}22.52 \\
\pm \\
9.23^{c}\end{array}$ \\
\hline $200-500$ & 39 & $\begin{array}{l}71.43 \pm \\
29.54^{\mathrm{bc}}\end{array}$ & $\begin{array}{l}186.46 \pm \\
61.33^{\mathrm{b}}\end{array}$ & $\begin{array}{l}23.70 \pm \\
5.51^{\mathrm{ab}}\end{array}$ & $9.26 \pm 4.37^{\mathrm{a}}$ & $\begin{array}{l}37.26 \\
\pm \\
9.52^{\mathrm{bc}}\end{array}$ \\
\hline $\begin{array}{l}500 \\
-1000\end{array}$ & 39 & $\begin{array}{l}101.40 \\
\pm \\
38.66^{\mathrm{ba}}\end{array}$ & $\begin{array}{l}262.64 \pm \\
41.77^{\mathrm{a}}\end{array}$ & $\begin{array}{l}25.14 \pm \\
3.42^{\mathrm{a}}\end{array}$ & $9.99 \pm 3.96^{\mathrm{a}}$ & $\begin{array}{l}46.01 \\
\pm \\
8.23^{\mathrm{a}}\end{array}$ \\
\hline $\begin{array}{l}1000 \\
-2000\end{array}$ & 36 & $\begin{array}{l}101.26 \\
\pm \\
31.45^{\text {ba }}\end{array}$ & $\begin{array}{l}277.87 \pm \\
43.18^{\mathrm{a}}\end{array}$ & $\begin{array}{l}23.83 \pm \\
5.31^{\mathrm{ab}}\end{array}$ & $\begin{array}{l}11.70 \pm \\
3.45^{\mathrm{a}}\end{array}$ & $\begin{array}{l}49.33 \\
\pm \\
0.86^{\mathrm{a}}\end{array}$ \\
\hline$>2000$ & 36 & $\begin{array}{l}125.44 \\
\pm \\
48.15^{\mathrm{a}}\end{array}$ & $\begin{array}{l}254.95 \pm \\
34.68^{\mathrm{a}}\end{array}$ & $\begin{array}{l}19.47 \pm \\
3.47^{\mathrm{bc}}\end{array}$ & $\begin{array}{l}12.00 \pm \\
4.23^{\mathrm{a}}\end{array}$ & $\begin{array}{l}53.02 \\
\pm \\
1.14^{\mathrm{a}}\end{array}$ \\
\hline \multirow[t]{2}{*}{$\begin{array}{l}\text { ANOVA } \\
\text { results }\end{array}$} & & $\begin{array}{l}F_{1,4}= \\
9.24\end{array}$ & $F_{1,4}=31.80$ & $F_{1,4}=10.05$ & $H_{4}=7.96$ & $\begin{array}{l}H_{4}= \\
39.28\end{array}$ \\
\hline & & $\begin{array}{l}p< \\
0.0001\end{array}$ & $p<0.0001$ & $p<0.0001$ & $p=0.0930$ & $\begin{array}{l}p< \\
0.0001\end{array}$ \\
\hline
\end{tabular}

Results of post-hoc tests performed on statistical analyses are indicated by lette indicating statistically significant differences. $n=$ number of analyses

${ }^{a}$ One-way ANOVA $(F)$, Tuckey post hoc test

${ }^{\mathrm{b}}$ Kruskal-Wallis ANOVA $(H)$, post hoc paired comparison test

Table 2

Pearson's linear correlation between elemental ( $\mathrm{C}$ and $\mathrm{N}$ ) and proximal (biochemical) components, and energy content of zooplankton in Kerguelen waters during the KEOPS2 survey

\begin{tabular}{|l|l|l|l|}
\hline $\begin{array}{l}\text { Chemical } \\
\text { components }\end{array}$ & $\mathbf{\%} \mathbf{C}$ & $\mathbf{\%}$ & $\mathbf{C} / \mathbf{N}$ \\
\hline \% lipids & $\begin{array}{l}r=0.754, p< \\
0.0001\end{array}$ & $\begin{array}{l}r=0.483, p< \\
0.0001\end{array}$ & $\begin{array}{l}r=0.662, p< \\
0.0001\end{array}$ \\
\hline \% proteins & $\begin{array}{l}r=0.917, p< \\
0.0001\end{array}$ & $\begin{array}{l}r=0.833, p< \\
0.0001\end{array}$ & $\begin{array}{l}r=0.489, p< \\
0.0001\end{array}$ \\
\hline $\begin{array}{l}r=\text { Pearson's linear correlation coefficient, } p=\text { probability value. Number of } \\
\text { samples, } n=186\end{array}$ & \multicolumn{2}{|l}{} \\
\hline
\end{tabular}

http://eproofing.springer.com/journals_v2/printpage.php?token=yVZa01xp-10rN8EC... 21/01/2019 


\begin{tabular}{|c|c|c|c|}
\hline $\begin{array}{l}\text { Chemical } \\
\text { components }\end{array}$ & $\% \mathrm{C}$ & $\% \mathrm{~N}$ & $\mathbf{C} / \mathbf{N}$ \\
\hline $\begin{array}{l}\% \text { soluble } \\
\text { carbohydrates }\end{array}$ & $\begin{array}{l}r=0.581, p< \\
0.0001\end{array}$ & $\begin{array}{l}r=0.594, p< \\
0.0001\end{array}$ & $\begin{array}{l}r=0.247, p= \\
0.0528\end{array}$ \\
\hline $\begin{array}{l}\% \text { insoluble } \\
\text { carbohydrates }\end{array}$ & $\begin{array}{l}r=0.448, p< \\
0.0001\end{array}$ & $\begin{array}{l}r=0.286, p= \\
0.0242\end{array}$ & $\begin{array}{l}r=0.374, p= \\
0.0028\end{array}$ \\
\hline Energy (J) & $\begin{array}{l}r=0.914, p< \\
0.0001\end{array}$ & $\begin{array}{l}r=0.712, p< \\
0.0001\end{array}$ & $\begin{array}{l}r=0.640, p< \\
0.0001\end{array}$ \\
\hline
\end{tabular}

Size fractions differed in their taxonomic composition (Table 3), which in turn influenced their elemental and biochemical compositions. The lowest size fraction (80-200 $\mu \mathrm{m}$ ) was generally composed of a mixture of phytoplankton, detritus and small zooplankton, but was sometimes dominated by diatoms (A3-2, E-4W), foraminifera (A3-1) or small copepods (R2) at some stations. All the other size fractions were dominated by copepods (Oithona similis, Microsetella rosea, Ctenocalanus citer, Calanus simillimus, Paraeuchaeta spp.), which differed in size and developmental stages in the different fractions, but varied slightly among stations (see Carlotti et al. 2015 for more details). The highest size fraction $(>2000 \mu \mathrm{m})$ was dominated by the copepod Rhincalanus gigas. This large fraction was also composed of different species of euphausiids, the amphipods Hyperia spp. and Themisto gaudichaudii, and large chaetognaths, pteropods and salps. The different groups of large zooplankton analyzed differed in their biochemical composition. Copepods (mainly R. gigas) presented by far the highest concentrations of lipids and proteins (Table 4), but the lowest concentration of carbohydrates ( $\mathrm{SC}+\mathrm{IC}=33.35 \mathrm{mg} \mathrm{g}^{-1} \mathrm{dw}$ compared to $37-45 \mathrm{mg} \mathrm{g}^{-1} \mathrm{dw}$ in the other groups). LIP concentration in copepods was $\times 1.6$ higher than in annelids, $\times 2.3$ higher than in euphausiids and $\times 3.1$ higher than in salps. Salps (mainly Salpa thompsoni) presented the lowest concentrations of LIP, PRO and SC, but the highest of IC. Euphausiids (mainly Thysanoessa sp.) and annelids (Tomopteris sp.) presented intermediate values, but did not differ statistically from copepods in PRO (Table 4). In terms of energy value, copepods were the most energy rich organisms analyzed $(15.3 \mathrm{~kJ}$ 
$\left.\mathrm{g}^{-1} \mathrm{dw}\right)$, followed by annelids $\left(10.4 \mathrm{~kJ} \mathrm{~g}^{-1} \mathrm{dw}\right)$, while salps were the least energetic $\left(6.3 \mathrm{~kJ} \mathrm{~g}^{-1} \mathrm{dw}\right)$ (Table 4).

Table 3

Mean composition of zooplankton size fractions sampled during the KEOPS2 survey in Kerguelen waters

\begin{tabular}{|c|c|}
\hline $\begin{array}{l}\text { Size fraction } \\
(\mu \mathrm{m})\end{array}$ & Groups of organisms \\
\hline $80-200$ & $\begin{array}{l}\text { Diatoms (40-90\%), Foraminifera, Detritus, Nauplii, } \\
\text { Copepods, Eggs, Radiolarians, Pteropods }\end{array}$ \\
\hline $200-500$ & $\begin{array}{l}\text { Copepods (50-95\%), Diatoms, Foraminifera, Pteropods, } \\
\text { Polychaetes, eggs }\end{array}$ \\
\hline $500-1000$ & $\begin{array}{l}\text { Copepods (70-95\%), Amphipods, Pteropods, Euphausiids, } \\
\text { Chaetognaths }\end{array}$ \\
\hline $1000-2000$ & $\begin{array}{l}\text { Copepods (70-95\%), Amphipods, Chaetognaths, Pteropods, } \\
\text { Euphausiids, Salps }\end{array}$ \\
\hline$>2000$ & $\begin{array}{l}\text { Copepods (60-95\%), Chaetognaths, Euphausiids, } \\
\text { Amphipods, Salps, Fish larvae, Annelids, Appendicularians, } \\
\text { Pteropods }\end{array}$ \\
\hline $\begin{array}{l}\text { The dominant } \\
\text { importance. T } \\
\text { groups is indic }\end{array}$ & $\begin{array}{l}\text { oups are indicated in bold characters in decreasing order of } \\
\text { range of visually estimated volume percentages of the main } \\
\text { ed into brackets }\end{array}$ \\
\hline
\end{tabular}

\section{Table 4}

Mean ( \pm SD) concentrations of lipids (LIP), proteins (PRO), soluble carbor insoluble carbohydrates (IC), percentages of organic carbon $(\% \mathrm{C})$ and nitrog energy content $(\mathrm{E})$ of some groups of large organisms $(>2000 \mu \mathrm{m})$

\begin{tabular}{|c|c|c|c|c|c|c|c|c|}
\hline Group & $\mathbf{n}$ & $\begin{array}{l}\mathrm{LIP}^{1} \\
\left(\mathrm{mg} \cdot \mathrm{g}^{-1}\right. \\
\mathrm{dw})\end{array}$ & $\begin{array}{l}\mathrm{PRO}^{1} \\
\left(\mathrm{mg} \cdot \mathrm{g}^{-1}\right. \\
\mathrm{dw})\end{array}$ & $\begin{array}{l}\mathrm{SC}^{1} \\
\left(\mathrm{mg} \cdot \mathrm{g}^{-1}\right. \\
\mathrm{dw})\end{array}$ & $\begin{array}{l}\mathrm{IC}^{2} \\
\left(\mathrm{mg} \cdot \mathrm{g}^{-1}\right. \\
\mathrm{dw})\end{array}$ & $\begin{array}{l}\% \mathrm{C}^{2} \\
\mathrm{dw}\end{array}$ & $\begin{array}{l}\% \mathrm{~N}^{2} \\
\mathrm{dw}\end{array}$ & $\boldsymbol{l}$ \\
\hline Copepods & 6 & $\begin{array}{l}242.56 \\
\pm \\
24.94^{\mathrm{a}}\end{array}$ & $\begin{array}{l}285.28 \\
\pm \\
15.49^{\mathrm{a}}\end{array}$ & $\begin{array}{l}15.49 \pm \\
2.25^{\mathrm{b}}\end{array}$ & $\begin{array}{l}17.86 \pm \\
3.83^{\mathrm{a}}\end{array}$ & $\begin{array}{l}55.12 \\
\pm \\
4.45^{\mathrm{a}}\end{array}$ & $\begin{array}{l}6.38 \pm \\
0.55^{\mathrm{b}}\end{array}$ & 1 \\
\hline Euphausiids & 9 & & & & & & & 1 \\
\hline
\end{tabular}

$n=$ number of samples analyzed. Results of post hoc tests performed on statisti are indicated by letters, different letters indicating statistically significant differ

${ }^{1}$ One-way ANOVA $(F)$, Tuckey post hoc test

${ }^{2}$ Kruskal-Wallis ANOVA $(H)$, post hoc paired comparison test 


\begin{tabular}{|c|c|c|c|c|c|c|c|c|}
\hline Group & $\mathbf{n}$ & $\begin{array}{l}\mathrm{LIP}^{1} \\
\left(\mathrm{mg} \cdot \mathrm{g}^{-1}\right. \\
\mathrm{dw})\end{array}$ & $\begin{array}{l}\mathrm{PRO}^{1} \\
\left(\mathrm{mg} \cdot \mathrm{g}^{-1}\right. \\
\mathrm{dw})\end{array}$ & $\begin{array}{l}\mathrm{SC}^{1} \\
\left(\mathrm{mg} \cdot \mathrm{g}^{-1}\right. \\
\mathrm{dw})\end{array}$ & $\begin{array}{l}\mathrm{IC}^{2} \\
\left(\mathrm{mg} \cdot \mathrm{g}^{-1}\right. \\
\mathrm{dw})\end{array}$ & $\begin{array}{l}\% C^{2} \\
d w\end{array}$ & $\begin{array}{l}\% \mathbf{N}^{2} \\
d w\end{array}$ & $\boldsymbol{I}$ \\
\hline & & $\begin{array}{l}104.57 \\
\pm \\
24.15^{b}\end{array}$ & $\begin{array}{l}279.48 \\
\pm \\
37.24^{\mathrm{a}}\end{array}$ & $\begin{array}{l}23.48 \pm \\
6.85^{b}\end{array}$ & $\begin{array}{l}13.92 \pm \\
4.48^{\mathrm{a}}\end{array}$ & $\begin{array}{l}51.39 \\
\pm \\
3.41^{\mathrm{ab}}\end{array}$ & $\begin{array}{l}11.21 \\
\pm \\
1.52^{\mathrm{a}}\end{array}$ & \\
\hline Annelids & 3 & $\begin{array}{l}147.92 \\
\pm \\
23.06^{b}\end{array}$ & $\begin{array}{l}249.37 \\
\pm \\
30.91^{\mathrm{a}}\end{array}$ & $\begin{array}{l}30.51 \pm \\
3.74^{\mathrm{a}}\end{array}$ & $\begin{array}{l}14.26 \pm \\
0.50^{\mathrm{a}}\end{array}$ & $\begin{array}{l}51.47 \\
\pm \\
0.72^{\mathrm{ab}}\end{array}$ & $\begin{array}{l}9.42 \pm \\
0.09^{\mathrm{ab}}\end{array}$ & $\begin{array}{l}1 \\
\cdots \\
\mathrm{t}\end{array}$ \\
\hline Salps & 12 & $\begin{array}{l}77.48 \pm \\
44.19^{c}\end{array}$ & $\begin{array}{l}132.51 \\
\pm \\
76.58^{b}\end{array}$ & $\begin{array}{l}17.89 \pm \\
8.30^{\mathrm{b}}\end{array}$ & $\begin{array}{l}21.75 \pm \\
20.50^{\mathrm{a}}\end{array}$ & $\begin{array}{l}42.20 \\
\pm \\
10.91^{\mathrm{b}}\end{array}$ & $\begin{array}{l}6.50 \pm \\
1.71^{\mathrm{b}}\end{array}$ & $\begin{array}{l}\ddot{6} \\
\ddot{l i} \\
\ddot{t} \\
\ddot{t}\end{array}$ \\
\hline \multirow[t]{2}{*}{$\begin{array}{l}\text { ANOVA } \\
\text { type }\end{array}$} & & $\begin{array}{l}F_{1,3}= \\
32.24\end{array}$ & $\begin{array}{l}H_{3}= \\
21.27\end{array}$ & $\begin{array}{l}H_{3}= \\
9.66\end{array}$ & $\begin{array}{l}H_{3}= \\
2.81\end{array}$ & $\begin{array}{l}H_{3}= \\
11.37\end{array}$ & $\begin{array}{l}H_{3}= \\
21.38\end{array}$ & $H$ \\
\hline & & $\begin{array}{l}p< \\
0.0001\end{array}$ & $\begin{array}{l}p< \\
0.0001\end{array}$ & $\begin{array}{l}p= \\
0.0217\end{array}$ & $\begin{array}{l}p= \\
0.4210\end{array}$ & $\begin{array}{l}p= \\
0.0099\end{array}$ & $\begin{array}{l}p< \\
0.0001\end{array}$ & $p$ \\
\hline
\end{tabular}

$n=$ number of samples analyzed. Results of post hoc tests performed on statisti are indicated by letters, different letters indicating statistically significant differ

${ }^{1}$ One-way ANOVA $(F)$, Tuckey post hoc test

${ }^{2}$ Kruskal-Wallis ANOVA $(H)$, post hoc paired comparison test

\section{Spatial variation in biochemical composition and energy content}

While station position were linked with temperature and salinity of water masses (cold and more salty at R2, A3-1 and A3-2 in AASW, and warm and less salty at F-L and TEW-8 in PFSW), chlorophyll $a$ concentration displayed a patchy distribution (Table 5). High Chl $a$ values $\left(>300 \mathrm{mg} \mathrm{m}^{-2}\right.$ ) were recorded at F-L and A3-2, low-Chl $a$ values $\left(<120 \mathrm{mg} \mathrm{m}^{-2}\right)$ at R2, A3-1, E-2, E-3 and E-4E, and intermediate values $\left(<120<\mathrm{Chl} a<200 \mathrm{mg} \mathrm{m}^{-2}\right)$ at the other stations. The mean biochemical composition of zooplankton, all fractions combined, differed significantly with station for LIP, SC and IC, but not for PRO (Table 5). The highest difference in component concentrations with space was observed for LIP $(\times 3$ from stations with the lowest to the highest values) and $\mathrm{IC}(\times 2.9)$, while the difference was 1.7 for PRO and 1.6 for SC. Mean concentrations of LIP peaked at station F-L and 
were the lowest at E-1 and E-2. PRO and SC concentrations were slightly higher at stations A3-1 and TEW-8, and IC concentration at F-L. During the Lagrangian survey, the mean LIP concentration of zooplankton was multiplied by 3.8 from E-1 to E-5, IC concentration by 1.9 and PRO concentration by 1.1, while SC concentration did not vary. However, while LIP concentration increased in time from E-1 to E-5, PRO concentration was much higher at station E-3 in the middle of the survey than at station E-5 at the end (Fig. 2). The total amount of energy $\left(\mathrm{E}_{\mathrm{T}}\right)$ provided by zooplankton biomass in the $0-250 \mathrm{~m}$ water column at each station during the KEOPS2 cruise was related to two main parameters, the biochemical composition and the biomass of zooplankton in each size fraction at station level. $\mathrm{E}_{\mathrm{T}}$ ranged from $26.35 \mathrm{~kJ} \mathrm{~m}^{-2} \mathrm{dw}$ at station E-4E to $116.73 \mathrm{~kJ} \mathrm{~m}^{-2} \mathrm{dw}$ at station F-L (mean $51.78 \pm 24.67 \mathrm{~J} \mathrm{~m}^{-2} \mathrm{dw}, n=186$ ). As illustrated in Fig. 3, $\mathrm{E}_{\mathrm{T}}$ was mainly related to the two largest size fractions (1000-2000 $\mu \mathrm{m}$ and $>2000 \mu \mathrm{m}$ ), which represented, respectively, an average of $23 \%$ and $59 \%$ of the total amount of energy provided by zooplankton in the 250 m-deep surface-water layer. Fraction $500-1000 \mu \mathrm{m}$ constituted $17 \%$ of $\mathrm{E}_{\mathrm{T}}$ and fraction $200-500 \mu \mathrm{m}$ only $1 \%$.

\section{Table 5}

Mean ( \pm standard deviation) concentrations of lipids (LIP), proteins (PRO), sol and insoluble carbohydrates (IC) in zooplankton $(0-250 \mathrm{~m})$ of Kerguelen durj and environmental parameters ( $\mp T$, $S S$, Chl $a$, T-group)

\begin{tabular}{|c|c|c|c|c|c|c|c|c|}
\hline Station & $n$ & Date & $\begin{array}{l}\text { T } \boldsymbol{T}^{4} \\
\left({ }^{\circ}\right. \\
\text { C) }\end{array}$ & $\mathbf{S} \boldsymbol{S}^{\mathbf{4}}$ & $\begin{array}{l}\text { Chl- } \\
a^{2} \\
(\mathrm{mg} \\
\left.\mathrm{m}^{-2}\right)\end{array}$ & T-group & $\begin{array}{l}\mathrm{LIP}^{3} \\
\left(\mathrm{mg} \cdot \mathrm{g}^{-1}\right. \\
\mathrm{dw})\end{array}$ & $\begin{array}{l}\text { PRO } \\
\text { (mg.ध } \\
\text { dw) }\end{array}$ \\
\hline $\mathrm{R} 2$ & 12 & $26 / 10 / 2011$ & 1.8 & 33.9 & 39 & $\mathrm{~T} 1$ & $\begin{array}{l}74.08 \pm \\
24.19 \\
\mathrm{ab}\end{array}$ & $\begin{array}{l}202.1 \\
\pm 81 . ! \\
\mathrm{a}\end{array}$ \\
\hline A3-1 & 12 & $20 / 10 / 2011$ & 1.8 & 34.0 & 106 & T3 & $\begin{array}{l}107.53 \\
\pm 10.91 \\
a\end{array}$ & $\begin{array}{l}307.4 \\
\pm 26 . \\
a\end{array}$ \\
\hline A3-2 & 15 & $16 / 11 / 2011$ & 1.8 & 34.0 & 372 & T3 & $\begin{array}{l}67.66 \pm \\
29.54 \\
\mathrm{ab}\end{array}$ & $\begin{array}{l}183.6 \\
\pm 82 . \\
a\end{array}$ \\
\hline TNS-7 & 15 & $22 / 10 / 2011$ & 2.2 & 33.9 & 150 & $\mathrm{~T} 1$ & $\begin{array}{l}65.58 \pm \\
31.19^{\mathrm{b}}\end{array}$ & $\begin{array}{l}177.0 \\
\pm 82.1 \\
\mathrm{a}\end{array}$ \\
\hline
\end{tabular}




\begin{tabular}{|c|c|c|c|c|c|c|c|c|}
\hline Station & $n$ & Date & $\begin{array}{l}\mathbf{F} \boldsymbol{T}^{4} \\
\left({ }^{\circ}\right. \\
\text { C) }\end{array}$ & $\mathbf{S} S^{\mathbf{4}}$ & $\begin{array}{l}\text { Chl- } \\
a^{2} \\
(\mathrm{mg} \\
\left.\mathrm{m}^{-2}\right)\end{array}$ & T-group & $\begin{array}{l}\mathrm{LIP}^{3} \\
\left(\mathrm{mg} \cdot \mathrm{g}^{-1}\right. \\
\mathrm{dw})\end{array}$ & $\begin{array}{l}\text { PRO } \\
(\mathrm{mg} \cdot \xi \\
\text { dw) }\end{array}$ \\
\hline E-1 & 15 & $30 / 10 / 2011$ & 2.3 & 33.9 & 130 & $\mathrm{~T} 2$ & $\begin{array}{l}50.35 \pm \\
21.51^{\mathrm{b}}\end{array}$ & $\begin{array}{l}190.9 \\
\pm 84 . \\
\mathrm{a}\end{array}$ \\
\hline E-2 & 15 & $01 / 11 / 2011$ & 2.3 & 33.9 & 100 & $\mathrm{~T} 2$ & $\begin{array}{l}56.79 \pm \\
24.20^{\mathrm{b}}\end{array}$ & $\begin{array}{l}201.3 \\
\pm 95 . \\
\mathrm{a}\end{array}$ \\
\hline E-3 & 15 & $04 / 11 / 2011$ & 2.3 & 33.9 & 80 & $\mathrm{~T} 2$ & $\begin{array}{l}119.35 \\
\pm 38.91 \\
\mathrm{a}\end{array}$ & $\begin{array}{l}251.0 \\
\pm 80 . \\
\mathrm{a}\end{array}$ \\
\hline E-4W & 15 & $11 / 11 / 2011$ & 2.4 & 33.9 & 135 & T3 & $\begin{array}{l}82.73 \pm \\
41.99 \\
\mathrm{ab}\end{array}$ & $\begin{array}{l}194.3 \\
\pm 94 . \\
\text { a }\end{array}$ \\
\hline$E-4 E$ & 12 & $13 / 11 / 2011$ & 2.4 & 33.9 & 105 & T5 & $\begin{array}{l}83.02 \pm \\
38.61 \\
\mathrm{ab}\end{array}$ & $\begin{array}{l}186.8 \\
\pm 74 . \\
\mathrm{a}\end{array}$ \\
\hline E-5 & 15 & $18 / 11 / 2011$ & 2.4 & 33.9 & 125 & T5 & $\begin{array}{l}118.87 \\
\pm 53.58 \\
\mathrm{a}\end{array}$ & $\begin{array}{l}213.4 \\
\pm 97 . \\
\mathrm{a}\end{array}$ \\
\hline TEW-4 & 15 & $01 / 11 / 2011$ & 2.4 & 33.9 & 170 & $\mathrm{~T} 2$ & $\begin{array}{l}86.06 \pm \\
59.15 \\
a b\end{array}$ & $\begin{array}{l}191.0 \\
\pm \\
113.4 \\
\mathrm{a}\end{array}$ \\
\hline TEW-8 & 15 & $02 / 11 / 2011$ & 3.6 & 33.7 & 185 & T1 & $\begin{array}{l}86.54 \pm \\
13.77 \\
a b\end{array}$ & $\begin{array}{l}279.1 \\
\pm 69 . \\
\mathrm{a}\end{array}$ \\
\hline F-L & 15 & $06 / 11 / 2011$ & 4.1 & 33.7 & 354 & T5 & $\begin{array}{l}151.73 \\
\pm 54.39 \\
\mathrm{a}\end{array}$ & $\begin{array}{l}200.8 \\
\pm 68 . \\
a\end{array}$ \\
\hline $\begin{array}{l}\text { ANOVA } \\
\text { results }\end{array}$ & & & & & & & $\begin{array}{l}H_{12}= \\
24.39 \\
p= \\
0.0180\end{array}$ & $\begin{array}{l}p= \\
0.405\end{array}$ \\
\hline
\end{tabular}

Stations are listed from south-west to north-east. Results of post hoc tests pe1 analyzes are indicated by letters, different letters indicating statistically signi number of biochemical analyzes. $T=$ sea surface temperature $\left({ }^{\circ} \mathrm{C}\right) . S=\operatorname{surfac}$ vertically integrated chlorophyll $a$ concentration from the surface to $200 \mathrm{~m}$. NOT in italics but in lower case -group = group of stations defined by Trull et a chemometric characteristics of phytoplankton

${ }^{1}$ Park et al. (2014) and Trull et al. (2015)

${ }^{2}$ Lasbleiz et al. (2014) 


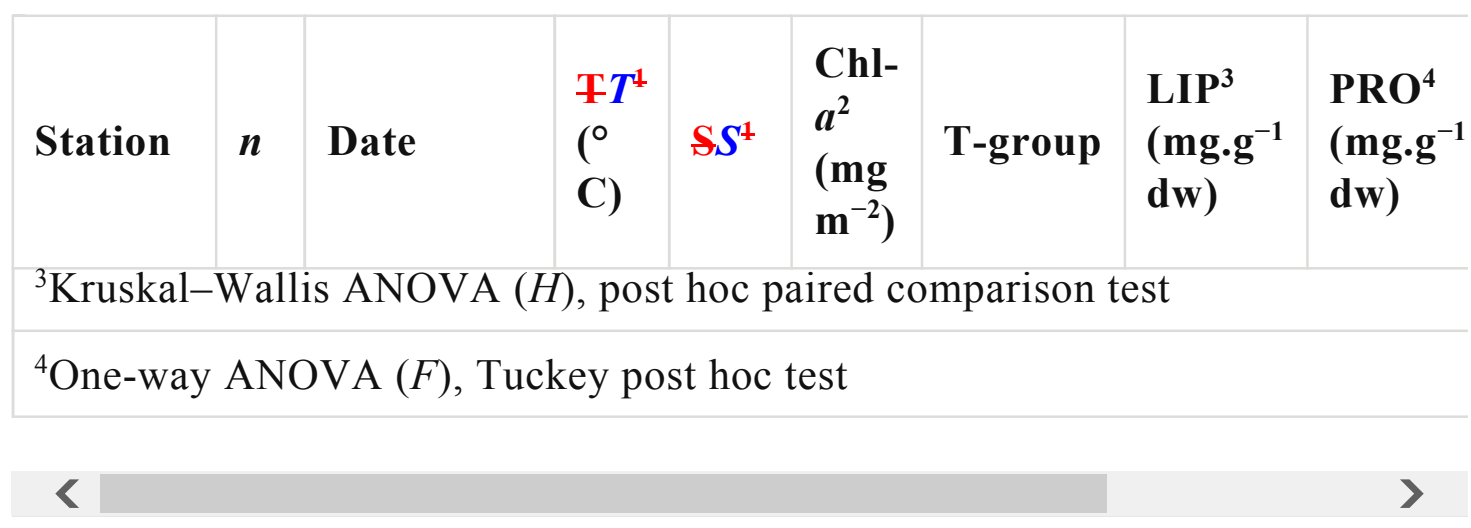

Fig. 2

Variation of lipid (top) and protein (bottom) percentages with size in zooplankton collected during the Lagrangian survey from stations E-1 to station E-5. The best-fitted curves follow the equation $y=\ln (x)-a$, where $y=$ compound percentage $(\%), x=$ fraction size, $a=$ constant depending on station 


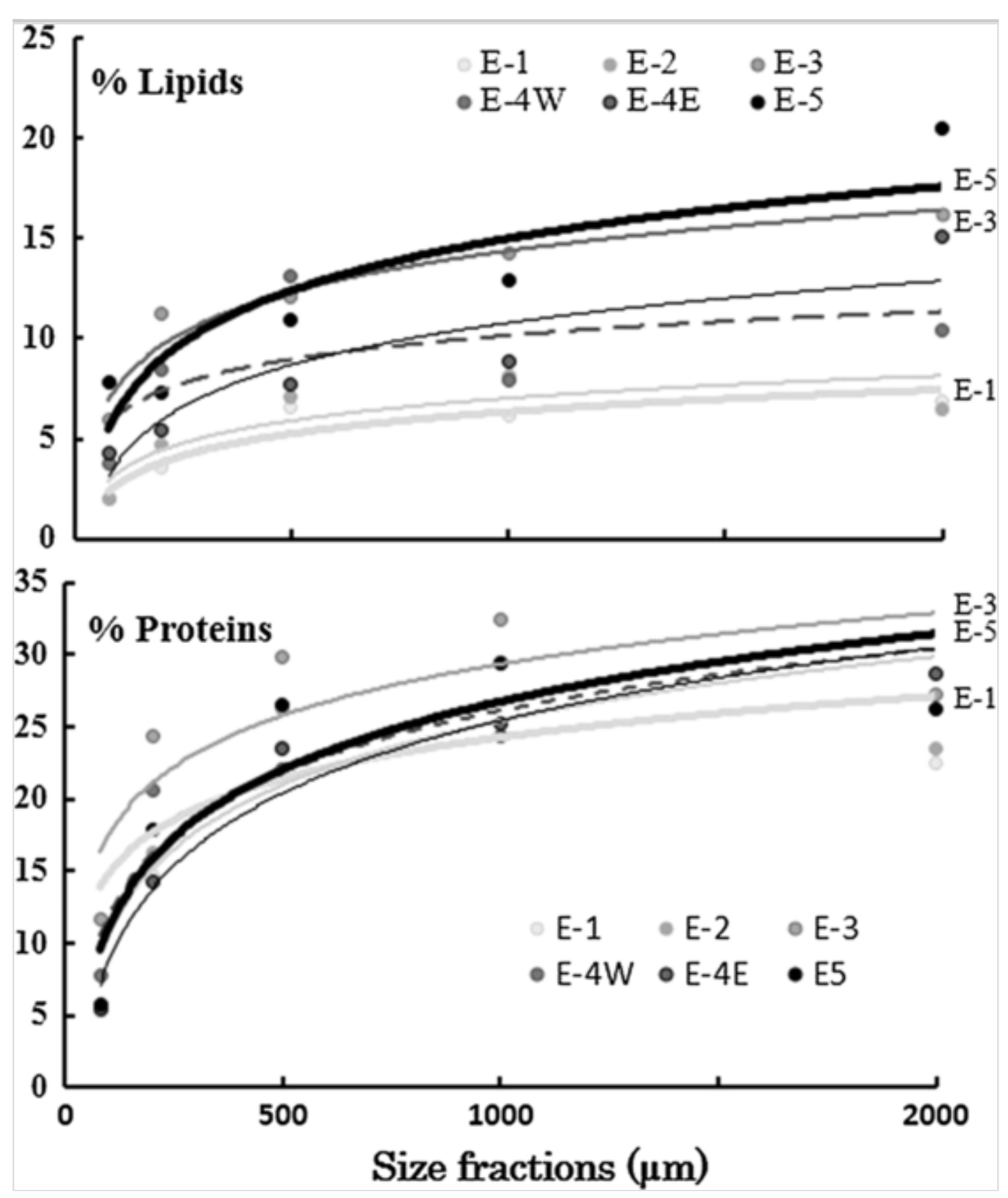

Fig. 3

Total energy content $\left(\mathrm{kJ} \mathrm{m}^{-2}\right)$ of zooplankton $>200 \mu \mathrm{m}$ in the $0-250 \mathrm{~m}$ water layer at the different stations sampled during KEOPS2 survey, with the relative importance of each size fraction in total energy 


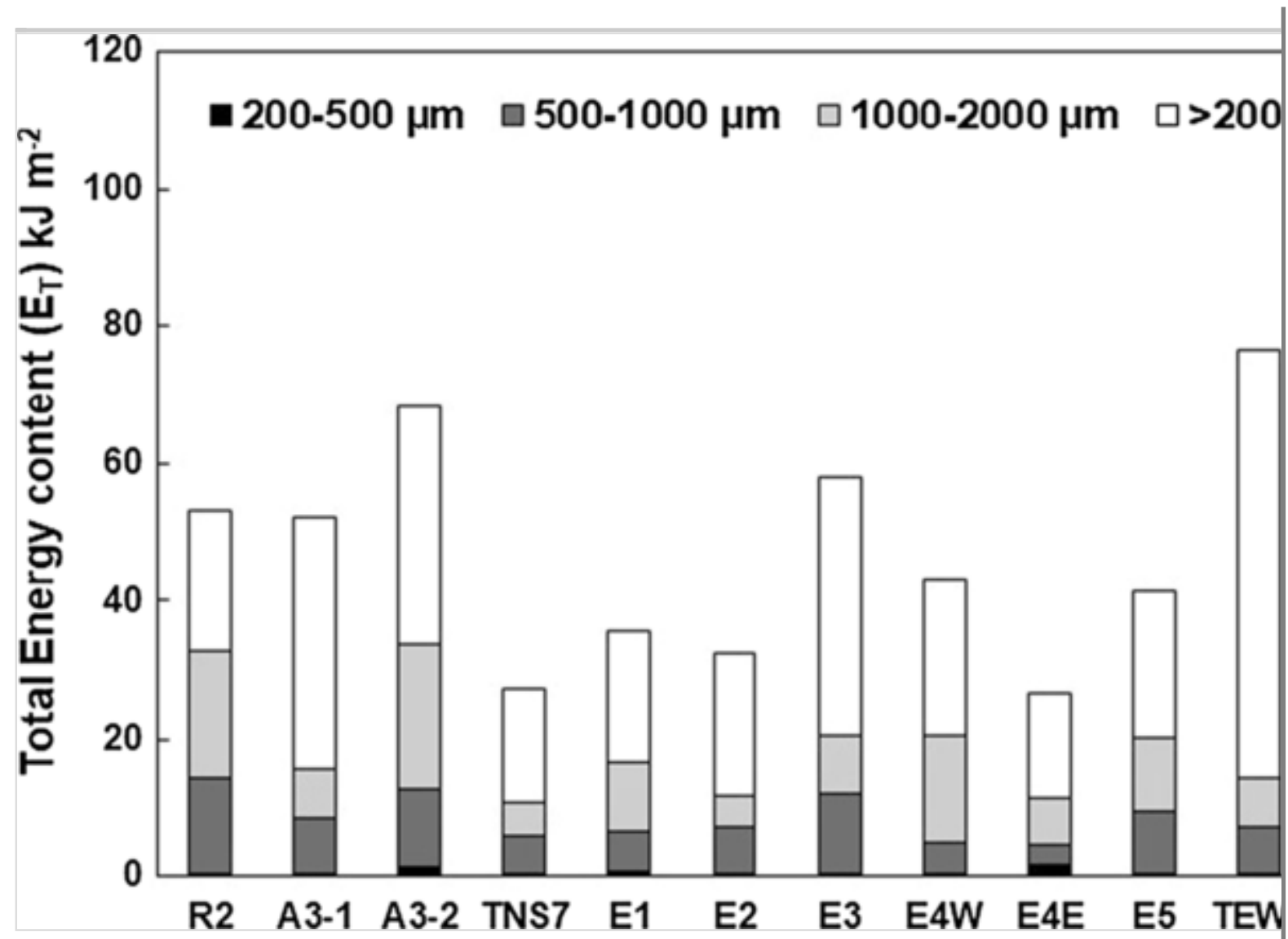

\section{Relative importance of factors}

The RDA explained $77.5 \%$ of the inertia of the biochemical composition of zooplankton (permutations $F$ test, $p<0.001$ ). The forward selection procedure of the RDA retained tten variables (three size classes, four stations, one T-group, sea surface temperature and chlorophyll $a$ concentration) explaining significantly the variation in the biochemical characteristics of plankton (Fig. 4). Date and salinity were not significant. The overall ordination was significant $(p<0.001)$. The RDA indicated that size was the most important factor in explaining the biochemical composition of zooplankton in Kerguelen waters (axis 1), followed by station, surface temperature and chlorophyll $a$ concentration (axis 2). The first axis explained $58.9 \%$ of the total variance and revealed the importance of size in accounting the biochemical and energy concentrations of zooplankton. E, SC, PRO, LIP, $\% \mathrm{C}$ and $\% \mathrm{~N}$ were all positively correlated with the largest plankton fraction $(>2000 \mu \mathrm{m})$ and negatively to the smallest fractions (80-200 $\mu \mathrm{m}$ and $200-500 \mu \mathrm{m}$ ). The second axis summarized $11.8 \%$ of the total variance and detected the importance of some stations, surface 
temperature and chlorophyll $a$ concentration in explaining differences associated with IC and LIP concentrations in zooplankton fractions as opposed to ISC concentration. Higher IC and LIP concentrations were positively associated with stations F-L and E5, higher sea surface temperature, and higher $\mathrm{Chl} a$ concentration. Higher IC concentration were also observed in T-group 2, but this factor was less important than $T\left({ }^{\circ} \mathrm{C}\right)$ and $\mathrm{Chl} a$. On the negative part of axis 2 , higher SC concentrations were associated with station TNS-7 and the 200-500 $\mu \mathrm{m}$ size class.

Fig. 4

Redundancy analysis (RDA): ordination diagram on the effects of environmental (station, date, $\mp T, \mathrm{~S} S, \mathrm{Chl} a$ and T-group) and morphological (size) factors on the biochemical and elemental characteristics of zooplankton (concentrations in macronutrients, energy, $\% \mathrm{C}$ and $\% \mathrm{~N}$ ) in Kerguelen waters 


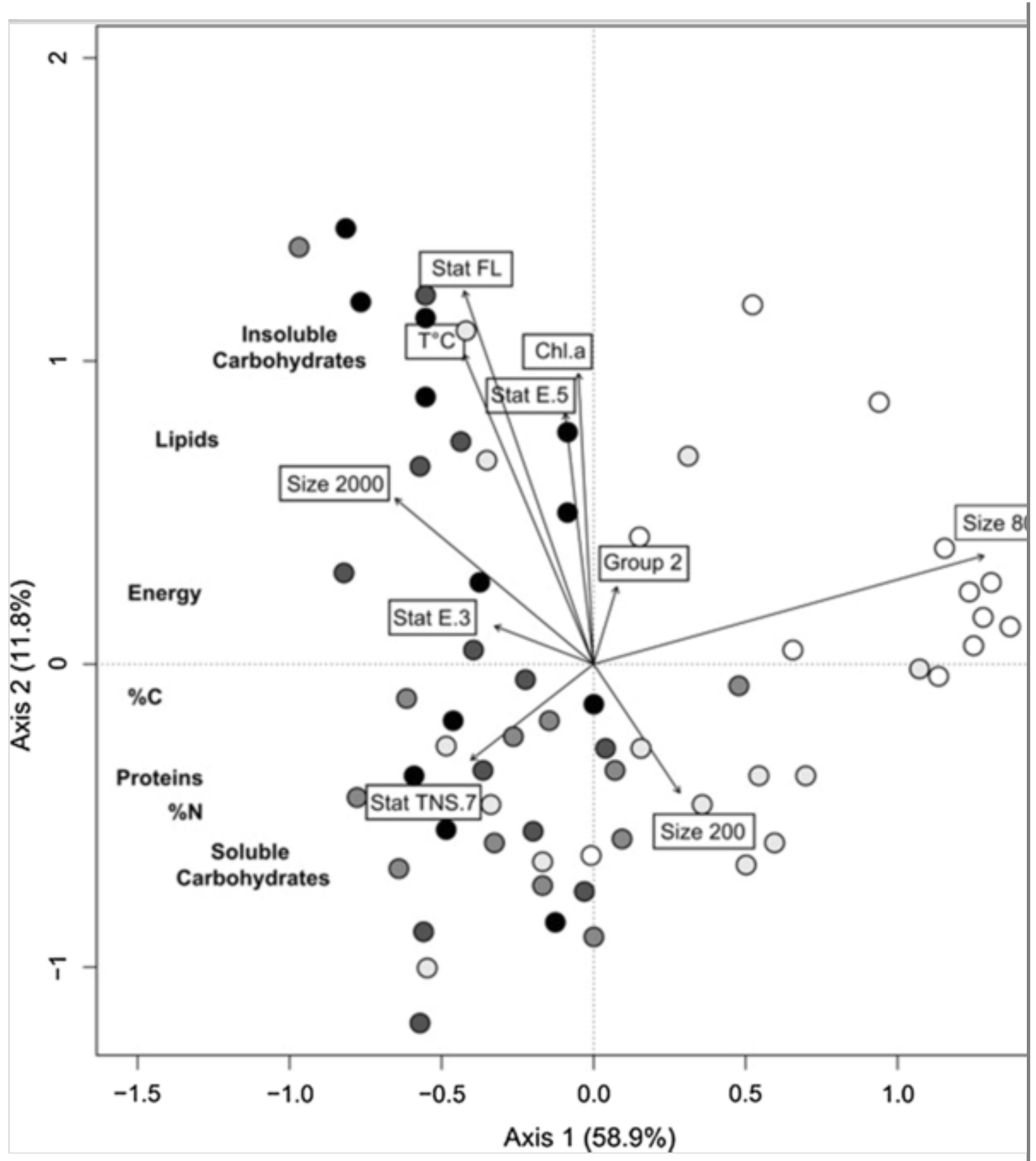

\section{Discussion}

The biochemical composition of zooplankton is an essential factor in the understanding of food web functioning and predator population dynamics, as zooplankton acts as 'a complex functional hub' in the transfer of organic matter between the lowest and the highest trophic levels in aquatic ecosystems (Banse 1995; Travers-Trolet et al. 2007). The main results of the present study indicated that (1) proteins formed the major biochemical component of zooplankton in Kerguelen waters $(22 \% \mathrm{dw})$, followed by lipids $(9 \% \mathrm{dw})$, while carbohydrates $(3 \% \mathrm{dw})$ 
represented only a weak percentage of organic constituents, (2) concentrations of all biochemical components increased with the size of zooplankton fractions, (3) the main part ( $>80 \%$ ) of the energy provided by zooplankton per station was due to large-sized organisms ( $>1000 \mu \mathrm{m}$ ), particularly copepods, which were the most energy-rich group analyzed, and (4) a high patchiness among stations was observed in the total energy provided by zooplankton, but the richest station was located in the polar front zone.

The dominance of proteins and the low percentage of carbohydrates in zooplankton are recorded in all oceanographic regions from tropical (Goswami et al. 1981; Arun Kumar et al. 2013) and temperate waters (Raymont et al. 1971; Mayzaud and Martin 1975) to polar zones (Percy and Fife 1981; Torres et al. 1994; Yun et al. 2015). Lipids present the most variable concentrations representing generally high percentages in zooplankton from Arctic and Antarctic waters (e.g. Donnelly et al. 1994; Falk-Petersen et al. 1999), and lower percentages in tropical zones (e.g. Arun Kumar et al. 2013; but see Cass et al. 2014 and Lee et al. 2006 for a review). However, the biochemical composition of zooplankton varies largely with species, size, ontogenic stage, site and season.

\section{Issues concerning zooplankton biochemical values}

The comparison between the numerous studies published on the biochemical composition of planktonic organisms and their energetic content is made difficult by differences in sampling methods (net mesh size, depth range), analytical methods, units in which results are expressed (most often in dry weight but also wet weight, ash-free dry weight, carbon weight), and the conversion factors used (see Postel et al. 2000; Yebra et al. 2017). In the present study, the residue (ashes and not recovered organic components) represents a high percentage of dry weight (44-84\% dw), depending on fraction size or group of organisms. This high residue percentage, particularly in the two smallest size fractions, could derive in part from large particulate aggregates and detritus (fecal pellets, diatom frustules, exuviae), abundant in the water column during the KEOPS2 survey (Jouandet et al. 2014). In bulk 
zooplankton samples collected with a Bongo net of $330 \mu \mathrm{m}$ mesh size, Bhat et al. (1993) recorded mean percentages of residue $(54-60 \% \mathrm{dw})$ close to those observed in our three largest size fractions $(58-60 \% \mathrm{dw})$. In the Mediterranean Sea, the residue percentage in similar zooplankton size fractions ranges from $64-84 \% \mathrm{dw}$ (C.T. Chen pers. comm.). When really quantified by combustion, ash percentages are highly variable among species or groups of organisms $(6-57 \% \mathrm{dw}$ in Ikeda et al. (1989), 7-31\% dw in Donnelly et al. (1993), 7-71\% dw in Donnelly et al. (1994), 6-70\% dw in Percy and Fife (199481), and 2-77\% in Postel et al. (2000)), being generally the lowest in copepods and the highest in tunicates and ctenophores. Clarke et al. (1992) recorded high-ash contents $(60-73 \% \mathrm{dw})$ in gelatinous zooplankton from the Southern Ocean, but only $12 \% \mathrm{dw}$ in the annelid Tomopteris. A mean ash content of $25.7 \% \mathrm{dw}\left(12.4 \_-35.5 \% \mathrm{dw}\right)$ was recorded by Postel et al. (2000) in different size classes of plankton from 55 to $>1000 \mu \mathrm{m}$. However, in addition to ash, there is still a proportion of non-explained organic matter, the sum of the biochemical components recovered plus ashes representing rarely $100 \%$ of the matter analyzed whatever the study and the environment. In effect, the analytical methods used may generate significant differences in component percentages, and do not quantify all the biochemical components present. For example, chitin, which may represent from 1 to $14 \% \mathrm{dw}$ of zooplankton (Mayzaud and Martin 1975; Donnelly et al. 1993; Torres et al. 1994), was not quantified in our study. Total lipids are generally measured following the Bligh and Dyer (1959) method, as we did. But Iverson et al. (2001) demonstrate that this method may underestimate lipid content by up to $50 \%$ when the percentage of lipids is $>2 \%$. When using the correction factor indicated by Iverson et al. (2001), the mean percentage of lipids in the zooplankton of Kerguelen Islands increases from 9 to $11 \% \mathrm{dw}$. The difference between the two methods increases with the size and concentration of zooplankton fraction, from $1.0 \%$ in the $80-200 \mu \mathrm{m}$ fraction to $2.7 \%$ in the fraction $>2000 \mu \mathrm{m}$. Proteins are most often quantified by the Lowry et al. (1951) method, but proteins do not represent all the nitrogen compounds of the organic matter (amino acids, chitin, etc.). From 12 to $42 \%$ dw of total nitrogen can be represented by non-protein nitrogen components in zooplankton (Mayzaud and Martin 1975; Donnelly et al. 1993). Thus, when the 
protein content is calculated in multiplying nitrogen percentages $(\% \mathrm{~N})$ by the classical 'Kjeldahl' conversion factor of 6.25 , much higher percentages of proteins are obtained. If we had used that kind of conversion factor, the mean percentage of proteins in Kerguelen zooplankton would have increased from 22 to $44 \% \mathrm{dw}$, with an increase difference of $19 \%$ for the $80-200 \mu \mathrm{m}$ fraction up to $27 \%$ for the fraction $>2000 \mu \mathrm{m}$. Similarly, the conversion factors used to transform the biochemical component concentrations into energy (calories and/or joules) differ among studies. These calculations may also differ from direct quantification of energy in a microbomb calorimeter. Conversion factors in caloric equivalents of organic constituents range from 9 $-9.5 \mathrm{kcal} \mathrm{g}^{-1}$ for lipids, from 4 to $5.7 \mathrm{kcal} \mathrm{g}^{-1}$ for proteins and from 4 to $4.2 \mathrm{kcal} \mathrm{g}^{-1}$ for carbohydrates (Clarke et al. 1992; Postel et al. 2000; Nageswara Rao and Ratna Kumari 2002). The conversion factors in joules used in the present paper $\left(35.6,21.4\right.$ and $17.2 \mathrm{~kJ} \mathrm{~g}^{-1}$ for lipids, proteins and carbohydrates, respectively) are those recommended by Postel et al. (2000) for zooplankton. Despite these problems in data comparison, our results agree with the general trends observed for biochemical composition variations with size and specific composition recorded elsewhere for zooplankton, and corresponded to minimum values.

\section{Effect of size and group composition on zooplankton biochemical composition}

Fraction size was the main factor explaining the biochemical composition of zooplankton in Kerguelen waters, with generally an increase in all component concentrations with size (Table 1, Fig. 4). Similar results are obtained at a specific level by different authors for all biochemical components (Guisande 2006) or lipids (Hagen and Auel 2001; Lee et al. 2006). The larger the organisms, the higher their concentrations in biochemical components. However, in the Mediterranean Sea, the highest concentrations are observed in the medium-size fractions of zooplankton (200-500 and 500-1000 $\mu \mathrm{m})$, with a decrease in concentrations in the largest fractions (1000-2000 and $>2000 \mu \mathrm{m})$ (C.T. Chen pers. comm.). Such a decrease is attributed to the dominance of gelatinous organisms (salps, siphonophores) in the largest size fractions. 
Concentrations in biochemical components, particularly lipids and proteins, differ considerably among species and groups of organisms (Percy and Fife 1981; Reinhardt and Van Vleet 1986; Torres et al. 1994; among many others). Therefore, the species or group composition of plankton size fractions greatly influences their biochemical composition. In this way, the higher PRO concentrations in all size fractions observed at station E-3, compared with the other stations E (see Fig. 2), could be related to a higher proportion of copepods (>90\%), amphipods and chaetognaths (all groups rich in proteins). Similarly, the 80-200 $\mu \mathrm{m}$ fraction contained higher concentrations of proteins and lipids (22.0\% and $7.3 \%$, respectively) at station R2, where this fraction was composed of small copepods, than at station A3-2 (5.3\% and $2.7 \%$ respectively) where this fraction was dominated by diatoms.

Among the few groups of organisms analyzed in Kerguelen waters, copepods showed the highest lipid, protein and energy content, followed by euphausiids, while the lowest values were recorded in salps. However, the $\% \mathrm{~N}$ was higher in euphausiids $(11 \% \mathrm{dw})$ than in copepods $(6 \% \mathrm{dw})$ (Table 4$)$. Similar results are observed in other studies, whatever the geographical region. Higher concentrations of proteins and lipids in copepods than in euphausiids are indicated by Goswami et al. (1981) in the Andaman Sea, who recorded similarly a higher $\% \mathrm{~N}(9 \% \mathrm{dw})$ in euphausiids than in copepods $(7 \% \mathrm{dw})$, perhaps due to a higher chitin content in euphausiids. Reinhardt and Van Vleet (1986) also found more lipids in copepods than in euphausiids of similar size in the Antarctic. In contrast, lower concentrations of biochemical components are generally recorded in gelatinous organisms, such as ctenophores, hydromedusae, tunicates and siphonophores (e.g. Goswami et al. 1981; Clarke et al. 1992; Donnelly et al. 1994), while protein concentration could be high in chaetognaths and pteropods (Mayzaud and Martin 1975; Percy and Fife 1981; Donnelly et al. 1994), as observed in Kerguelen waters.

Both $\% \mathrm{C}$ and $\% \mathrm{~N}$ were more strongly correlated with proteins than with lipids, while energy was more strongly correlated with $\% \mathrm{C}$ than with $\%$ $\mathrm{N}(r=0.914$ vs $0.712, n=186$, respectively) (see Table 2$)$. The high dependence of energy on organic carbon content is well known for 
aquatic organisms (Salonen et al. 1976). Similar results were also observed in Antarctic krill by Färber-Lorda et al. (2009), with the best correlation found between joules and carbon. As expected, the $\mathrm{C} / \mathrm{N}$ ratio was better correlated with lipids $(r=0.662)$ than with other components, a well-documented relation in zooplankton (e.g. Postel et al. 2000; Färber-Lorda et al. 2009).

\section{Variations of biochemical composition among stations}

Wide spatial and temporal variations in the biochemical composition of zooplanktonic organisms are reported in the literature (Raymont et al. 1971; Donnelly et al. 1993; Nageswara Rao and Ratna Kumari 2002). Major variations are particularly observed in polar regions, where lipid storage is an important seasonal phenomenon (Percy and Fife 1981; Ward et al. 1996; Lee et al. 2006; Mayzaud et al. 2011). Wide variations in the biochemical composition of size-fractionated zooplankton among stations were also recorded in Kerguelen waters during the onset of the spring bloom (Table 5). Two types of component varied particularly with ontogeny and time, lipids as involved in energy storage, and insoluble carbohydrates as involved in cellular membrane formation. While LIP were significantly positively related to fraction size (Table 1), IC concentrations were significantly related to the abundance of organisms ( $r=0.55, p=0.034, n=62)$ for fractions between $200 \mu \mathrm{m}$ and $2000 \mu \mathrm{m}$, mainly composed of copepods (Table 3 ). The similar mean concentration of proteins among stations (Table 5) could reflect the fact that proteins are the main structural component in zooplankton growth (Carlotti et al. 1993), which fluctuates less than non-structural components, such as some lipid classes linked to energy storage (Falk-Petersen et al. 1999; Hagen and Auel 2001; Cass et al. 2014). Variation in biochemical component concentrations among stations reflected the patchiness in composition and population response of zooplankton communities to the spring bloom, as stations were sampled at different dates and bloom conditions. The patchiness of rapidly changing biomass at the onset of the spring bloom in this region, evidenced for both phytoplankton (Lasbleiz et al. 2014; Trull et al. 2015) and zooplankton communities (Carlotti et al. 2015), was thus also observed in their biochemical composition. 
The data obtained during the Lagrangian survey between station E-1 and E-5 (eddy in oceanic AASW) gave some information on the biochemical changes in zooplankton as the bloom developped (Fig. 2). Carlotti et al. (2015) observed a strong increase in zooplankton abundance during this survey, with the cumulative contribution of various larval stages of the dominant copepods and euphausiids. Protein concentrations $(\times 1.1$ between E-1 and E-5) responded less than lipids $(\times 3.8$ in the same time) to the increase in zooplankton abundance. As proteins were mostly involved in structural weight (Carlotti et al. 1993), while lipids were the most efficient component for non-structural energy storing, this could suggest an increase in feeding activity of zooplankton during the development of the bloom.

Strong seasonality in total lipid content has been well studied in Arctic and Antarctic planktonic organisms, particularly in crustaceans, such as copepods, euphausiids and amphipods, that have to respond to food shortage and diapause for winter survival, then to reproduction and ontogeny during phytoplankton bloom (Percy and Fife 1981; Reinhard and van Vleet 1986; Mayzaud et al. 2011). The energy content of planktonic organisms varies widely with season according to trophic resource variations and zooplankton growth (e.g. Percy and Fife 1981; Donnelly et al. 1994; Mayzaud et al. 2011). An increase in both lipid and energy content of zooplankton as the season progresses, reflecting an increase in feeding at all trophic levels, is observed in all organisms, but modulated by species physiology (Lee et al. 2006; Kattner et al. 2007; Cass et al. 2014). Lipid storage is particularly important in herbivorous copepods experiencing winter diapause in deep waters such as Rhincalanus gigas (Ward et al. 1996; Hagen and Auel 2001; Lee et al. 2006), which was the most abundant copepod species in the largest plankton size fraction in Kerguelen waters ( $>75 \%$ in number) (Carlotti et al. 2015). However, as emphasized by many authors, as proteins are the major organic components, they may also serve as metabolic reserve in zooplankton organisms, in addition to lipids, but such a role may be more important in temperate and tropical waters (Arun Kumar et al. 2013) than in polar regions. 


\section{Role of zooplankton energy in food web functioning}

The energy content of zooplankton in Kerguelen waters increased with the size of the organisms, the three largest and biomass-dominant fractions analyzed (500-1000 $\mu \mathrm{m}, 1000-2000 \mu \mathrm{m}$ and $>2000 \mu \mathrm{m})$ having a mean energy value of $\geq 10 \mathrm{~kJ} \mathrm{~g}^{-1}$. A mean energy value of $12.5 \mathrm{~kJ} \mathrm{~g}^{-1}$ for total zooplankton (> $300 \mu \mathrm{m}$ ) has been recorded by Goswami et al. (1981) in the Andaman Sea, but only $7.5 \mathrm{~kJ} \mathrm{~g}^{-1}$ by Arun Kumar et al. (2013) in the same region, and $10.6 \mathrm{~kJ} \mathrm{~g}^{-1}$ by Bhat et al. (1993) in the Arabian Sea. The energy content of Kerguelen organisms were within the range of those recorded in other studies for similar groups, with high values recorded in copepods and annelids, and low values in salps (Table 6). The energy content (E) of zooplankton was positively and significantly related both to lipid and protein concentrations of the organisms, the highest correlation being naturally observed with lipids due to their high energy content (nearly twice that of proteins). However, being the dominant biochemical components, proteins constituted an average of 55\% of the energy provided by zooplankton in Kerguelen, while lipids represented $38 \%$ and carbohydrates only $7 \%$.

Table 6

Mean energy content of some groups of zooplankton organisms $\left(\mathrm{kJ} \mathrm{g}^{-1} \mathrm{dw}\right)$

\begin{tabular}{|l|l|l|l|l|l|}
\hline Reference & Site & Copepods & Euphausiids & Annelids & Salps \\
\hline This study & $\begin{array}{l}\text { Kerguelen } \\
\text { Islands }\end{array}$ & 15.3 & 10.3 & 11.4 & 6.3 \\
\hline $\begin{array}{l}\text { Goswami } \\
\text { et al. } \\
(1981)\end{array}$ & Andaman Sea & 17.2 & 13.9 & - & 6.9 \\
\hline $\begin{array}{l}\text { Percy and } \\
\text { Fife (1981) }\end{array}$ & Baffin Island & - & 24.3 & 18.8 & - \\
\hline $\begin{array}{l}\text { Clarke et } \\
\text { al. (1992) }\end{array}$ & South Georgia & - & - & 16.3 & 5.4 \\
\hline $\begin{array}{l}\text { Donnelly } \\
\text { et al.(1994) }\end{array}$ & Weddell Sea & 14.4 & & 9.2 & - \\
\hline $\begin{array}{l}\text { Torres et } \\
\text { al. (1994) }\end{array}$ & Weddell Sea & - & 17.5 & - & - \\
\hline$<$ & & & & \} $\\
{\hline}$
\end{tabular}




\begin{tabular}{|l|l|l|l|l|l|}
\hline Reference & Site & Copepods & Euphausiids & Annelids & Salps \\
\hline $\begin{array}{l}\text { Chen (pers. } \\
\text { comm.) }\end{array}$ & $\begin{array}{l}\text { Mediterranean } \\
\text { Sea }\end{array}$ & & & \\
\hline
\end{tabular}

Zooplankton grazing on phytoplankton corresponded not only to the OM transfer up into the food web but also to a crucial qualitative leap of increasing nutritive and energetic density of this matter. Differences in biochemical composition and energy content between the 80-200 $\mu \mathrm{m}$ size class at stations where it was dominated by diatoms (representative of 'phytoplankton') and those of the $>500 \mu \mathrm{m}$ size classes (representative of 'zooplankton') illustrated this phenomenon (Fig. 5). The shift from phyto to zooplankton enhanced by four $(\times 4.2)$ the energy density of each gram of organic matter $\left(2.42 \mathrm{~kJ} \mathrm{~g}^{-1} \mathrm{dw}\right.$ and $10.14 \mathrm{~kJ} \mathrm{~g}^{-1} \mathrm{dw}$, respectively), providing a more nutritive and energetic food source to fulfill the metabolic requirements of upper level predators. Such a qualitative leap was linked to an increase in protein and lipid concentrations in zooplankton, compared to those of phytoplankton (Fig. 5). By comparison, an increase in OM energy density of only $\times 1.3$ was observed from euphausiids to myctophids using the mean energy value for these fish species $\left(13.6 \mathrm{~kJ} \mathrm{~g}^{-1}\right)$ recorded by Lea et al. (2002) in Kerguelen waters (Fig. 5). Similarly, Waluda et al. (2012) observe an increase of $\times 1.5$ in OM energy value from krill to planktivorous fish such as myctophids in Subantarctic waters (4.65. $\mathrm{kJ} \mathrm{g}^{-1} \mathrm{ww}$ and $6.99 \mathrm{~kJ} \mathrm{~g}^{-1} \mathrm{ww}$, respectively).

\section{Fig. 5}

Increase in energy density $\left(\mathrm{E}\right.$, in $\mathrm{kJ} \mathrm{g}^{-1}$ ) of the organic matter from phytoplankton to zooplankton, and from euphausiids to myctophids, in Kerguelen waters. The proportions of energy provided by proteins, lipids and carbohydrates are indicated for each group of organisms. Data for phytoplankton, zooplankton and euphausiids are issued from this study, and data for myctophids are issued from Lea et al. (2002) in Kerguelen waters 


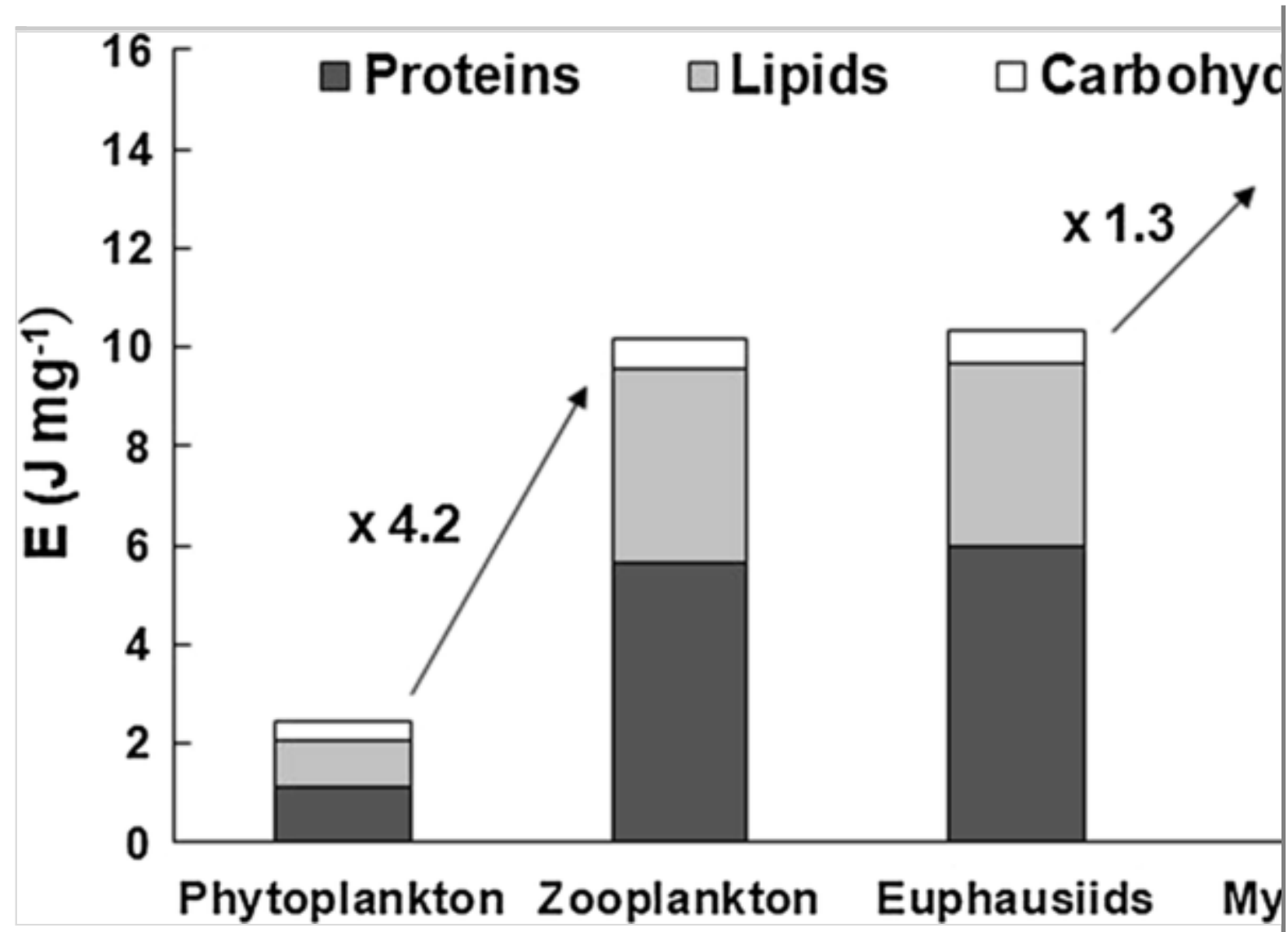

The two largest zooplankton fractions $(1000-2000 \mu \mathrm{m}$ and $>2000 \mu \mathrm{m})$ analyzed, rich in lipids and proteins, represented $80 \%$ on average of the biomass (Carlotti et al. 2015) and $82 \%$ of the energy provided by zooplankton in the $0-250 \mathrm{~m}$ water layer east of the Kerguelen Islands during the KEOPS2 survey (Fig. 3). The fraction 500-1000 $\mu \mathrm{m}$ completed the biomass and energy contribution to the whole zooplankton, particularly at the most productive stations (F-L, E-3, A3-2), while the fraction 200-500 $\mu$ m contributed only for a few $\%$ both in biomass and energy of the whole zooplankton. Among zooplankton organisms, large copepods presented the highest concentrations in lipids and the highest energy content (Table 4). Many studies on food web structure in the Southern Ocean evidence the importance of meso- and macrozooplankton, either as a direct food source for a wide array of zooplankton consumers (e.g. amphipods, euphausiids, fish and seabirds), or indirectly, amphipods, euphausiids and fish being themselves forage species consumed by high-level predators (Pruvost et al. 2005; Hindell et al. 2011). Euphausiids, while primarily grazing on phytoplankton, are also able to feed on a large size range of zooplankton, mainly copepods (from $100 \mu \mathrm{m}$ to several $\mathrm{mm}$ ), 
and the varying importance of the copepod contribution in their diets is nowadays recognized (Schmidt et al. 2014; Sogawa et al. 2017). The high energy values of the mesozooplankton size fractions above $200 \mu \mathrm{m}$ found in our study, mainly boosted by copepod energy value, could represent an essential role for the trophic requirement of some euphausiid species, as shown in other ecosystems (Huenerlage and Buchholz 2013; Schmidt and Atkinson 2016; Sogawa et al. 2017). Myctophid fishes, which are an important trophic link between zooplankton and high-level predators, mainly feed on large $(>1 \mathrm{~mm})$ copepods ( $>50 \%$ by number), then on euphausiids, hyperiid amphipods and pteropods (Pakhomov et al. 1996; Cherel et al. 2010). Planktonic crustaceans, particularly large copepods, euphausiids and amphipods, also constitute the main prey of many species of seabirds, such as petrels and penguins that breed in the area of the Kerguelen and Crozet Islands (Ridoux 1994; Guinet et al. 1996; Bocher et al. 2002; Cherel et al. 2005).

The total energy content provided by zooplankton east of Kerguelen Islands presented a patchy distribution (Fig. 3), as also emphasized for chlorophyll $a$ concentration, and phytoplankton and zooplankton abundances (Lasbleiz et al. 2014; Carlotti et al. 2015). The highest lipid and total energy contents were recorded at F-L, a station located in the PFSW polar front zone (Park et al. 2014). This could suggest a role of oceanic front in providing more nutritive food resources for upper-level consumers. However, more sampling in this zone would have been necessary to support this hypothesis. The influence of oceanic front in increasing zooplankton abundance, biomass and grazing activity has been well documented in the Southern Atlantic (Mayzaud et al. 2007; Richoux 2011; Lopes et al. 2015). Recently, Woodson and Litvin (2014), incorporating hydrological patchiness and front into current fishery models, show the crucial role of oceanic fronts in sustaining global marine fishery production. In the southern oceans, long-term tracking of different marine birds and mammals indicates that front zones constitute preferential foraging zones for many of them (Bost et al. 2009).

Thus, the high abundance, large size and high-energy content of zooplankton of Kerguelen waters could explain why it constitutes a 
major food resource for the numerous fish, seabirds and marine mammals of this region. Further studies on the temporal and spatial variation in the biochemical composition of zooplankton in this region would be needed to better understand how the physical parameters and the development of the phytoplankton bloom influence the energy transfer along the food web. However, information provided here could be still used to better estimate the energetic budget of trophic compartments in this region. The functional role of zooplankton involves not only accelerating OM transfer but also increasing its energy performance, a pivotal role that should be better consider in modeling studies. Modeling of food web functioning would be more realistic if based on energy units rather than matter content $(\mathrm{C}, \mathrm{N}, \mathrm{P})$. However, few data giving the energy value of planktonic community by size classes are still available. AQ2

AQ3

\section{Acknowledgements}

The authors thank S. Blain, project coordinator, B. Quéguiner chief scientist on board, and Captain B. Lassiette and the crew of the R/V Marion Dufresnes. Special thanks are due to M.P. Jouandet for her help in sample collection, M.F. Fontaine for biochemical analyzes, to L. Guillou and P. Richard (LIENSs Laboratory, La Rochelle University) for $\mathrm{C}$ and $\mathrm{N}$ analyzes, and to $\mathrm{M}$. Paul, a native English speaker, for English correction. This work was supported by the French Research program of INSU-CNRS LEFE - CYBER ("Les enveloppes fluides et l'environnement-Cycles biogéochimiques, environnement et resources"), the French ANR (Agence Nationale de la Recherche, ANR-10-BLAN-0614 of SIMI-6 program, and ANR09-CEXC-006-01 to M. Zhou and F. Carlotti), LABEX OT-MED (No. ANR-11-LABX-0061), the French CNES (Centre National d'Etudes Spatiales) and the French Polar Institute IPEV (Insitut Polaire Paul-Emile Victor). The project leading to this publication has received funding from European FEDER Fund under Project 116639417. Many thanks are addressed to the three reviewers for their constructive and helpful comments on an earlier version of the manuscript. 


\section{Compliance with ethical standards}

Conflict of interest The authors declare that they have no conflict of interest.

\section{References}

Arun Kumar M, Padmavati G, Anandavelu I (2013) Biochemical composition and calorific value of zooplankton from the coastal waters of South Andaman. Proc Int Acad Ecol Environ Sci 3:278 $-287$

Banse K (1995) Zooplankton: pivotal role in the control of ocean production. ICES J Mar Sci 52:265-277

Barnes C, Maxwell D, Reuman DC, Jennings S (2010) Global patterns in predator-prey size relationships reveal size dependency of trophic transfer efficiency. Ecology 91:222-232

Beukema JJ (1997) Caloric values of marine invertebrates with an emphasis on the soft parts of marine bivalves. Oceanogr Mar Biol Ann Rev 35:387-414

Bhat KL, Sreepada RA, Ansari ZA (1993) Biochemical composition of zooplankton from the northern Arabian sea. Pak J Mar Sci 2:17 $-22$

Blain S, Quéguiner B, Trull T (2008) The natural iron fertilization experiment KEOPS (Kerguelen Ocean Plateau Compared Study): an overview. Deep-Sea Res Pt II 55:559-565

Blanchard JL, Heneghan RF, Everett JD, Trebilco R, Richardson AJ (2017) From bacteria to whales: Using functional size spectra to model marine ecosystems. Trends Ecol Evol 32:174-186

Bligh EG, Dyer WJ (1959) A rapid method of total lipid extraction and purification. Can J Physiol Pharmacol 37:911-917 
Bocher P, Cherel Y, Labat JP, Mayzaud P, Razouls S, Jouventin P (2001) Amphipod-based food web: Themisto gaudichaudii caught in nets and by seabirds in Kerguelen waters, southern Indian Ocean. Mar Ecol Prog Ser 223:261-276

Bocher P, Cherel Y, Alonzo F, Razouls S, Labat JP, Mayzaud P, Jouventin P (2002) Importance of the large copepod Paraeuchaeta antarctica (Giesbrecht, 1902) in coastal waters and the diet of seabirds at Kerguelen, Southern Ocean. J Plankton Res 24:1317 $-1333$

Bost CA, Cotté C, Bailleul F, Cherel Y, Charrassin JB, Guinet C, Ainley DG, Weimerskirch H (2009) The importance of oceanic fronts to marine birds and mammals of the southern oceans. J Mar Syst 78:363-376

Carlotti F, Krause M, Radach G (1993) Growth and development of Calanus finmarchicus related to the influence of temperature: experimental results and conceptual model. Limnol Oceanogr $38: 1125-1134$

Carlotti F, Giske J, Werner F (2000) Modelling zooplankton dynamics. In: Harris R, Wiebe P, Lenz J, Skjoldal HR, Huntley M (eds) ICES zooplankton methodology manual. Academic Press, San Diego, pp 571-667

Carlotti F, Jouandet MP, Nowaczyk A, Harmelin-Vivien M, Lefèvre D, Richard P, Zhu Y, Zhou M (2015) Mesozooplankton structure and functioning during the onset of the Kerguelen bloom during Keops2 survey. Biogeosciences 12:4543-4563

Cass CJ, Daly KL, Wakeham SG (2014) Assessment of storage lipid accumulation patterns in eucalanoid copepods from the eastern tropical Pacific Ocean. Deep-Sea Res Pt I 93:117-130

Cherel Y, Bocher P, Trouvé C, Weimerskirch H (2002) Diet and feeding ecology of blue petrels Halobaena caerulea at Iles Kerguelen, Southern Indian Ocean. Mar Ecol Prog Ser 228:283-299 
Cherel Y, Fontaine C, Richard P, Labat JP (2010) Isotopic niches and trophic levels of myctophid fishes and their predators in the Southern Ocean. Limnol Oceanogr 55:324-332

Cherel Y, Bost CA, Guinet C, Weimerskirch H (2005) Feeding habits of seabirds and marine mammals of the Kerguelen Archipelago. In: Palomares MLD, Pruvost P, Pitcher TJ, Pauly D (eds) Modeling Antarctic marine ecosystems. Fisheries Centre Research Reports, 13(7). Fisheries Centre, University of British Columbia, Vancouver, pp 31-36

Clarke A, Holmes LJ, Gore DJ (1992) Proximate and elemental composition of gelatinous zooplankton from the Southern Ocean. J Exp Mar Biol Ecol 155:55-68

Donnelly J, Stickney DG, Torres JJ (1993) Proximate and elemental composition and energy content of mesopelagic crustaceans from the Eastern Gulf of Mexico. Mar Biol 115:469-480

Donnelly J, Torres JJ, Hopkins TL, Lancraft TM (1994) Chemical composition of Antarctic zooplankton during austral fall and winter. Polar Biol 14:171-183

Dubois M, Gilles KA, Hamilton JK, Reber PA, Smith F (1956) Colorimetric method for determination of sugar and related substances. Anal Chem 28:350-356

Falk-Petersen S, Sargent JR, Loenne OJ, Timofeev S (1999) Functional biodiversity of lipids in Antarctic zooplankton: Calanoides acutus, Calanus propinquus, Thysanoessa macrura and Euphausia crystallorophias. Polar Biol 21:37-47

Färber-Lorda J, Gaudy R, Mayzaud P (2009) Elemental composition, biochemical composition and caloric value of Antarctic krill. Implications in energetics and carbon balances. J Mar Syst 78:518 $-524$ 
Frederiksen M, Edwards M, Richardson AJ, Halliday NC, Wanless S (2006) From plankton to top predators: bottom-up control of a marine food web across four trophic levels. J Anim Ecol 75:1259 $-1268$

García-Comas C, Sastri AR, Ye L, Chang CY, Lin FS, Su MS, Gong GC, Hsieh CH (2016) Prey size diversity hinders biomass trophic transfer and predator size diversity promotes it in planktonic communities. Proc R Soc B 283:2015-2129

Goswami SC, Rao TSS, Matondkar P (1981) Biochemical composition of zooplankton from the Andaman Sea. Indian J Mar Sci 10:296-300

Guinet C, Cherel Y, Ridoux V, Jouventin P (1996) Consumption of marine resources by seabirds and seals in Crozet and Kerguelen waters: Changes in relation to consumer biomass. 1962-1985. Antarct Sci 8:23-30

Guisande C (2006) Biochemical fingerprints in zooplankton. Limnetica 25:369-376

Hagen W, Auel H (2001) Seasonal adaptations and the role of lipids in oceanic zooplankton. Zoology 104:313-326

Hansen B, Bjornsen PK, Hansen PJ (1994) The size ratio between planktonic predators and their prey. Limnol Oceanogr 39:395-403

Hindell MA, Bost CA, Charrassin JB, Gales N, Lea MA, Goldsworthy S, Page B, Robertson G, Wienecke W, O'Toole M, Guinet C (2011) Foraging habitats of top predators, and areas of ecological significance, on the Kerguelen plateau. In: Duhamel G, Welsford D (eds) The Kerguelen Plateau: marine ecosystem and fisheries. Société d'Ichtyologie, Paris, pp 203-215

Huenerlage K, Buchholz F (2013) Krill of the northern Benguela Current and the Angola-Benguela frontal zone compared: 
physiological performance and short-term starvation in Euphausia hanseni. J Plankton Res 35:337-351

Ikeda T, Skjoldal HR (1989) Metabolism and elemental composition of zooplankton from the Barrents Sea during early Arctic summer. Mar Biol 100:173-183

Iverson SJ, Lang SLC, Cooper MH (2001) Comparison of the Bligh and Dyer and Folch methods for total lipid determination in a broad range of marine tissue. Lipids 36:1283-1287

Jouandet MP, Jackson G, Carlotti F, Picheral M, Stemmann L, Blain S (2014) Rapid formation of large aggregates during the spring bloom of Kerguelen Island: observations and model comparisons. Biogeosciences 11:4949-4993

Kattner G, Hagen W, Lee RF, Campbell R, Deibel D, Falk-Petersen S, Graeve M, Hansen BW, Hirche HJ, Jónasdottir SH, Madsen ML, Mayzaud P, Müller-Navarra D, Nichols PD, Paffenhöfer GA, Pond D, Saito H, Stübing D, Virtue P (2007) Perspectives on marine zooplankton lipids. Can J Fish Aquat Sci 64:1628-1639

Lasbleiz M, Leblanc K, Blain S, Ras J, Cornet-Barthaux V, Hélias Nunige S, Quéguiner B (2014) Pigments, elemental composition (C, $\mathrm{N}, \mathrm{P}$, and $\mathrm{Si}$ ), and stoichiometry of particulate matter in the naturally iron fertilized region of Kerguelen in the Southern Ocean.

Biogeosciences 11:5931-5955

Lea MA, Nichols PD, Wilson G (2002) Fatty acid composition of lipid-rich myctophids and mackerel icefish (Chamsocephalus gunnari) - Southern Ocean food-web implications. Polar Biol 25:843 $-854$

Lee RF, Hagen W, Kattner G (2006) Lipid storage in marine zooplankton. Mar Ecol Progr Ser 307:273-306

Legendre L, Legendre P (2012) Numerical ecology, 3rd edn. Elsevier, New York 
Lopes RM, Marcolin CR, Brandini FP (2015) Influence of oceanic fronts on mesozooplankton abundance and grazing during spring in the south-western Atlantic. Mar Freshw Res 67:626-635

Lowry O, Rosenbrough RJ, Farr L, Randall R (1951) Protein measurements with the Folin phenol sulfuric acid method. Water Res 7:741-746

Mayzaud P, Martin JLM (1975) Some aspects of the biochemical and mineral composition of marine plankton. J Exp Mar Biol Ecol $17: 297-310$

Mayzaud P, Laureillard J, Merien D, Labat JP (2007) Zooplankton nutrition, storage and fecal lipid composition in different water masses associated with the Agulhas and Subtropical Fronts. Mar Chem 107:202-213

Mayzaud P, Lacombre S, Boutoute M (2011) Seasonal and growth stage changes in lipid and fatty acid composition in the multigeneration copepod Drepanus pectinatus from Iles Kerguelen. Antarct Sci 23:3-17

Nageswara Rao I, Ratna Kumari R (2002) Biochemical composition of zooplankton from Visakhapatnam harbour waters, east coast of India. Indian J Mar Sci 31:125-129

Pakhomov EA, Perissinotto R, McQuaid CD (1996) Prey composition and daily rations of myctophid fishes in the Southern Ocean. Mar Ecol Prog Ser 134:1-14

Park YH, Durand I, Kestenare E, Rougier G, Zhou M, d'Ovidio F, Cotté C, Lee JH (2014) Polar Front around the Kerguelen Islands: An up-to-date determination and associated circulation of surface/subsurface waters. J Geophys Res Oceans 119:6575-6592

Pauthenet E, Roquet F, Madec G, Guinet C, Hindell M, McMahon CR, Harcourt R, Nerini D (2018) Seasonal meandering of the Polar 
Front upstream of the Kerguelen plateau. Geophys Res Lett. https://doi.org/10.1029/2018GL079614

Percy JA, Fife FJ (1981) The biochemical composition and energy content of Arctic marine macrozooplankton. Arctic 34:307-313

Postel L, Fock H, Hagen W (2000) Biomass and abundance. In: Harris R, Wiebe P, Lenz J, Skjoldal HR, Huntley M (eds) ICES zooplankton methodology manual. Academic, San Diego, pp 82-192

Pruvost P, Duhamel G, Palomares MLD (2005) An ecosystem model of the Kerguelen Islands'EEZ. In: Palomares MLD, Pruvost $P$, Pitcher TJ, Pauly D (eds) Modeling Antarctic marine ecosystems. Fisheries Centre Research Reports, 13(7). Fisheries Centre, University of British Columbia, Vancouver, pp 40-46

Raymont JEG, Srinivasagam RT, Raymont JKB (1971) The biochemical composition of Euphausia superba. J Mar Biol Assoc UK 51:581-588

Reinhardt SB, VanVleet ES (1986) Lipid composition of twenty-two species of Antarctic midwater zooplankton and fish. Mar Biol 91:149 $-159$

Richoux N (2011) Trophic ecology of zooplankton at a frontal transition zone: fatty acid signatures at the subtropical convergence, Southern Ocean. J Plankton Res 33:491-505

Ridoux V (1994) The diets and dietary segregation of seabirds at the subantarctic Crozet Islands. Mar Ornithol 22:1-192

Salonen K, Sarvala J, Hakala I, Viljanen ML (1976) The relation of energy to organic carbon in aquatic invertebrates. Limnol Oceanogr 21:724-730

Schmidt K, Atkinson A (2016) Feeding and food processing in Antarctic krill (Euphausia superba Dana). In: Siegel V (ed) Biology 
and Ecology of Antarctic krill, Euphausia superba Dana 1850. Springer, Cham, pp 175-224

Schmidt K, Atkinson A, Pond DW, Ireland LC (2014) Feeding and overwintering of Antarctic krill across its major habitats: The role of sea ice cover, water depth, and phytoplankton abundance. Limnol Oceanogr 59:17-36

Sogawa S, Sugisaki H, Tadokoro K, Ono T, Sato E, Shimode S, Kikuchi T (2017) Feeding habits of six species of euphausiids (Decapoda: Euphausiacea) in the northwestern Pacific Ocean determined by carbon and nitrogen stable isotope ratios. J Crust Biol $37: 29-36$

Torres JJ, Donnelly J, Hopkins TL, Lancraft TM, Aarset AV, Ainley DG (1994) Proximate composition and overwintering strategies of Antarctic micronektonic Crustacea. Mar Ecol Prog Ser 113:221-232

Travers-Trolet M, Shin YJ, Jennings S, Cury P (2007) Towards endto-end models for investigating the effects of climate and fishing in marine ecosystems. Prog Oceanogr 75:751-770

Trull TW, Davies DM, Dehairs F, Cavagna AJ, Lasbleiz M, Laurenceau-Cornec EC, d'Ovidio F, Planchon F, Leblanc K, Quéguiner B, Blain S (2015) Chemometric perspectives on plankton community responses to natural iron fertilization over and downstream of the Kerguelen Plateau in the Southern Ocean. Biogeosciences 12:1029-1056

Waluda CM, Hill SL, Peat HJ, Trathan PN (2012) Diet variability and reproductive performance of macaroni penguins Eudyptes chrysolophus at Bird Island, South Georgia. Mar Ecol Prog Ser 466:261-274

Ward P, Shreeve RS, Cripps GC (1996) Rhincalanus gigas and Calanus simillimus: lipid storage patterns of two species of copepod in the seasonally ice-free zone of the Southern Ocean. J Plankton Res 18:1439-1454 
Woodson CB, Litvin SY (2014) Ocean fronts drive marine fishery production and biogeochemical cycling. Proc Natl Acad Sci USA 112:1710-1715

Yebra L, Kobari T, Sastri AR, Gusmão F, Hernández-León S (2017) Advances in biochemical indices of zooplankton production. Adv Mar Biol 76:157-240

Yun MS, Lee DB, Kim BK, Kang JJ, Lee JH, Yang EJ, Park WG, Chung KH, Lee SH (2015) Comparison of phytoplankton macromolecular compositions and zooplankton proximate compositions in the northern Chukchi Sea. Deep Sea Res Pt II 120:82-90 